NBSIR 76-985

\begin{tabular}{l} 
A Computer Model to Determine \\
Low Cost Techniques to Comply \\
with the Privacy Act of 1974 \\
\hline
\end{tabular}

Dr. Robert C. Goldstein

Dr. Henry H. Seward

D. P. Management Corporation

1 Militia Drive

Lexington, Massachusetts 02173

February 1976

Final

Prepared for the

Systems and Software Division Institute for Computer Sciences and Technology National Bureau of Standards 
NBSIR 76-985

\section{A COMPUTER MODEL TO DETERMINE LOW COST TECHNIQUES TO COMPLY WITH THE PRIVACY ACT OF 1974}

Dr. Robert C. Goldstein

Dr. Henry H. Seward

D. P. Management Corporation

1 Militia Drive

Lexington, Massachusetts 02173

February 1976

Final

Prepared for the

Systems and Software Division

Institute for Computer Sciences and Technology

National Bureau of Standards

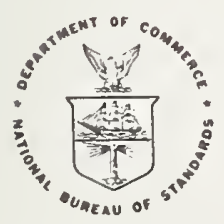

U.S. DEPARTMENT OF COMMERCE, Rogers C.B. Morton, Secrotary James A. Baker, III, Under Secretary Dr. Betsy Ancker-Johnson, Assistant Secretary for Science and Technology NATIONAL BUREAU OF STANDARDS, Ernest Ambler, Acting Director 

The work presented on these pages represents, to our knowledge, a uniquely concrete and objective approach to evaluating some of the economic considerations resulting from the Privacy Act of 1974 (PL 93579). By using a computer model to simulate the cost impact of the Act's requirements, one can determine the differences resulting from alternative approaches to implementing the mandated safeguards.

The computer model and the methodology it represents can be altered and modified to reflect changing circumstances and different record keeping systems. The publication of this work reflects our desire to improve the model by soliciting comments and suggestions from those involved in dealing with the task of complying with the law. By acting as a central collection point for these comments, NBS can provide a pool of knowledge that will aid all agencies. 

Table of Contents

Section

Page

1. Introduction 1

2. Program Documentation 3

2.1 Program Execution 3

2.2 The Input 3

2.3 Format Statements 3

2.4 Data Input 4

2.5 The Output 7

Appendix 1 Computer Program Listing 16

$\begin{array}{ll}\text { Appendix } 2 \text { Glossary } & 27\end{array}$

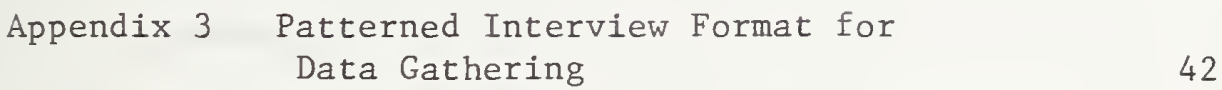

FIGURE 1 Program Format Statements 4

FIGURE 2 Input Record Layout 7

FIGURE 3 Combined Output Report 10

FIGURE 4 Example of Output Section 非1 11

FIGURE 5 Example of Output Section 非2 12

FIGURE 6 Summary Output Report 15 



\subsection{INTRODUCTION}

This document contains a complete description of the steps necessary to run the DPM Cost of Privacy Model along with a description of the computer program. The computer model has been developed for the National Bureau of Standards by the D. P. Management Corporation of Lexington, Massachusetts, and is made available for federal agency use. In a companion NBS document, entitled Methodology for Evaluating Alternative Technical and Information Management Approaches to Privacy Requirements, the algorithms contained in this model are explained. The interested reader is advised to review this document.

The document is divided into three sections. The first describes general system requirements for running the program. The second describes how to input information to the model. And the third describes the output and how to interpret it.

In reading the output, the user should be aware that computer output run on "batch" processing with output on high speed printers is usually limited to 132 characters of printing across a page.

This is the form of output familiar to most people. For many terminals, on the other hand, the number of characters is usually limited to 72 characters per line. Such output looks very similar to a typed page of information. The output of this model may be adapted quite easily to either form of processing.

An important aspect of any model is its ability to be used for comparing numerous results based on various assumptions. Although the Cost of Privacy Model is not unique in this aspect, it is extremely 
important for the user to be aware of this capability, because of the very obvious subjective aspects of some of the input. As the user becomes familiar with the input and output from the model, specific attention should be placed on how to manipulate the data and thus how to achieve reliable results.

The model is not overly complex and the input and output are fairly simple to understand. Because the 1974 Privacy Act is new, however, certain input values are difficult to determine. Examples are:

- The number of data subjects who will make inquiries.

- The amount of executive time necessary to handle data disputes.

- Programming time to develop software to handle Privacy Act requirements.

Undoubtedly, as experience with the law and information interchange between affected agencies grows, additional precision can be gained. 


\subsection{PROGRAM DOCUMENTATION}

\section{1 PROGRAM EXECUTION}

Because there is so much variation between different computer facilities, no attempt will be made at explaining exactly how to make the model operational on a specific processor. A skilled programmer familiar with a unique computer facility having the minimal equipment and the basic capabilities described below should have no trouble getting the model to execute.

\section{Language - ANSI Fortran \\ Length - 531 Records \\ Core Requirements - Less than 35K Bytes}

The program 1isting has been included as Appendix 1.

\subsection{THE INPUT}

\subsubsection{General Comments}

The data for this model is put on 36 successive cards, each of which is a maximum of 80 characters. This section will explain what is found on each of the records, how each one is formatted, and how this data is "read" into the program.

\subsubsection{Types of Input}

To make the program as generally useful as possible, it was written to accept input from standard 80 column cards. Installations with online computing capability may wish to change the input and output unit specifications to enable terminal or file-oriented input and output.

\subsection{FORMAT STATEMENTS IN THE PROGRAM}

There are six different input formats within the model. Format 8005 is used for reading, in succession, the abbreviations for each of 
the presently programmed twenty compliance steps*. Three formats (8001-8003) are used to read in various information about the system under study. 8004 and 8005 are used to read several additional parameters into the model. Format 8006 is used to input data concerning number of runs, copies, and whether a sumary output is desired. Figure 1 is a list of these six formats as they appear in the program.

\section{Figure 1 - Program Format Statements}

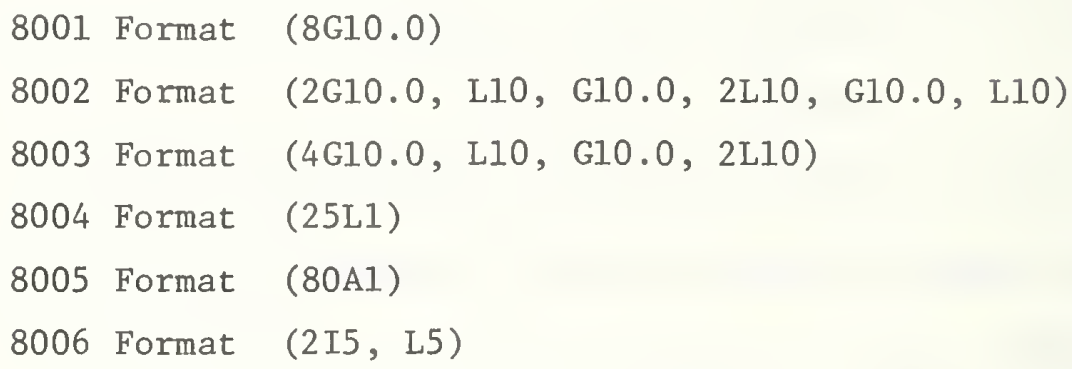

\subsection{DATA INPUT}

\subsubsection{Compliance Step Names (8005 Format, Records 非1-20)}

Presently, there are twenty different compliance steps (or techniques) analyzed by the model. Since any or all of these can occur in the output, abbreviated names for each must be supplied. Normally, these names would be written directly into the program. However, since ANSI Fortran does not include a convenient mechanism for specifying alphabetic constants, these names are included as part of the program's input. Also, they are left as input so the analyst may vary them to

*Compliance steps are steps an agency must take to comply with the 1974 Privacy Act. (See NBS Technical Note, A Methodology for Evaluating Alternative Technical and Information Management Approaches to Privacy Requirements, August 1975, for discussion of specific steps. 
identify alternative techniques. Once they are set up, they remain constant for all runs of the program. Each name can contain letters, numbers, periods, or any other appropriate symbol. The following names are currently in the input:

1. Data supply obligation notification

2. Consent for additional use

3. Check usage authorization

4. Usage log maintenance

5. Record existence notification

6. Record existence inquiry

7. Record uses inquiry

8. Data accuracy

9. Additional data

10. Data accuracy inquiry

11. Subject claim storage

12. Subject claim dissemination

13. Retroactive claim dissemination

14. Record transmission

15. Consent to transfer data

16. Legal process notification

17. Physical security

18. User training

19. System assurance

20. Public notice

Remember, although not all twenty compliance steps need be assessed, twenty names must still be in the input.

\subsubsection{Execution Instructions (8006 Format, Record \#21)}

The execution instruction card contains three instructions which dictate how many iterations of the model will be made, how many copies of the output will be printed, and whether a summary output will be produced. The number of iterations should be in the first five spaces, the number of outputs should be in the next five spaces. A "T" (true) should be placed in colum 15 if a summary output is desired. 


\subsubsection{Date of Computer Run (8005 Format, Record 非2)}

The date of the computer run or some other run identification is put on the next (22nd) record. It must be less than 21 characters long and start in column 1.

2.4.4 Title of Computer Run (8005 Format, Record 非3)

An identification title is put on the next (23rd) record. It has a maximum length of 80 characters. This identifier can be used to keep successive runs of the model separately identified. 2.4.5 Logic Gates (8004 Format, Record 非4)

The next record (24th) contains 20 logical true (T) or false (F) gates. These gates should be set to "true" (T) if the compliance technique is to be analyzed or "false" (F) if it is not. They are in sequential order, as listed on page 5 , in fixed format in columns 1 through 20 of the record.

\subsubsection{System Attributes (Formats 8001-3, Records 非25-36)}

The 1 ast 12 records contain 75 separate pieces of information pertinent to the computer system under study for compliance with the Privacy Act. Each of the 12 records contains specific information, which must be placed in specific locations on the record. Each of the 12 records is listed in Figure 2, in order, showing the exact location for each piece of data. The acronyms used in this figure are explained in Appendix 2, the glossary, and/or Appendix 3, the patterned interview. 
FIGURE 2 - INPUT RECORD LAYOUTS

Input

Record 非

Record Columns

\begin{tabular}{lllllllll} 
& 1-10 & $11-20$ & $21-30$ & $31-40$ & $41-50$ & $51-60$ & $61-70$ & 71-80 \\
\cline { 2 - 5 } & NSUB & NREC & TRRT & TVAL & NCHK & SREC & NUSR & \\
26 & NUST & NCLK & CLINT & NTRN & ONLINE & ISTR & NRPR & DMS \\
27 & SANS & NFRM & QFRM & NLPN & ISRC & TCPU & XG & XS \\
28 & SMRS & FMRS & SCPR & RTPR & SDTR & FDTR & SYSP & APPP \\
29 & EXEC & CLER & AUDT & & & & & \\
30 & SADD & SCLM & SLOG & IWUL & IINT & TADT & & \\
31 & HADT & HFRM & HSPL & HJDG & HPCL & HGSN & HINQ & HGRD \\
32 & HUID & HCAD & HVER & HTRC & HTRU & & & \\
33 & PADT & PUID & PNOT & PACC & PCVD & PDCL & PLOG & PRET \\
34 & PINT & PWAC & & & & & & \\
35 & NNUS & FRPT & KLOG & KSEC & PRGMNT & FOBJ & FREI & FRUI \\
36 & FDAI & FRDS & & & & & &
\end{tabular}

2.5 THE OUTPUT

2.5.1 General Comment

This section explains how to read the model's two outputs. Samples of the two output reports have been included in Figure 4 and Figure 5 . Figure 3 illustrates the method of combining the two reports for analysis. 2.5.2 Definition of Terms Used on the Output

TOTAL EXPENSES - The section of the report which lists total conversion (CONV) and total annual (ANNUAL) costs.

CONVERSION EXPENSES - The section of the report which lists conversion costs by resource.

ONGOING EXPENSES-The section of the report which lists annual costs by resource.

COMP. TECH. - Compliance Technique

CONV. TOTALS - Conversion Totals 


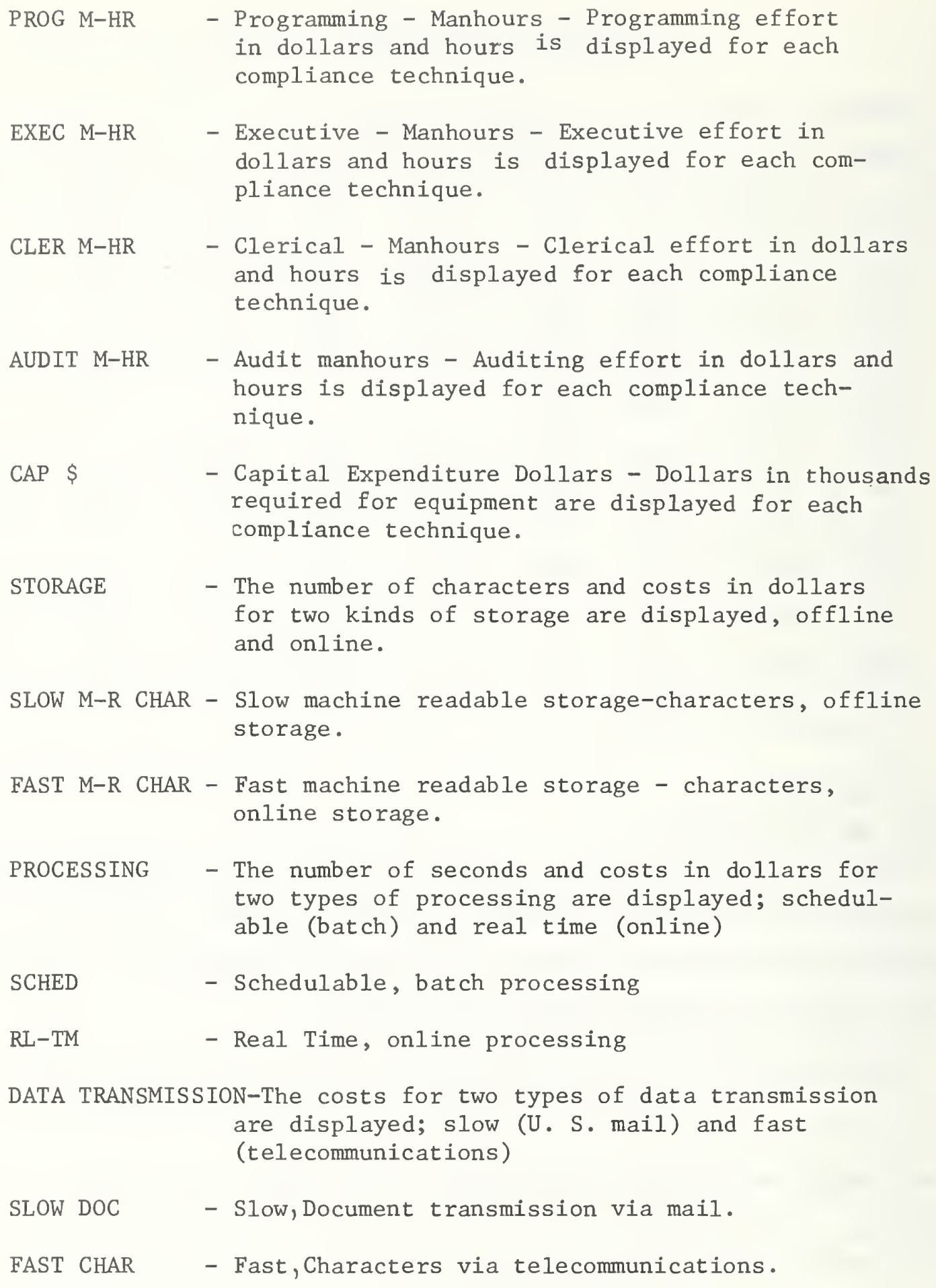

SLOW M-R CHAR - Slow machine readable storage-characters, offline storage.

FAST M-R CHAR - Fast machine readable storage - characters, online storage.

PROCESSING - The number of seconds and costs in dollars for two types of processing are displayed; schedulable (batch) and real time (online)

SCHED - Schedulable, batch processing

RL-TM - Real Time, online processing

DATA TRANSMISSION-The costs for two types of data transmission are displayed; slow (U. S. mail) and fast (telecommunications)

SLOW DOC - Slow, Document transmission via mail.

FAST CHAR - Fast, Characters via telecommunications. 


\subsubsection{Full Output Report}

\subsubsection{General Comment}

The full output is divided into two sections and should be obtained from a 132 character output device. The first section contains compliance technique cost totals and a breakout of the conversion (nonrecurring) expenses. The second section contains the ongoing (recurring) expenses. The most efficient way to read the report is to place the two sections side by side (See Figure 3). 


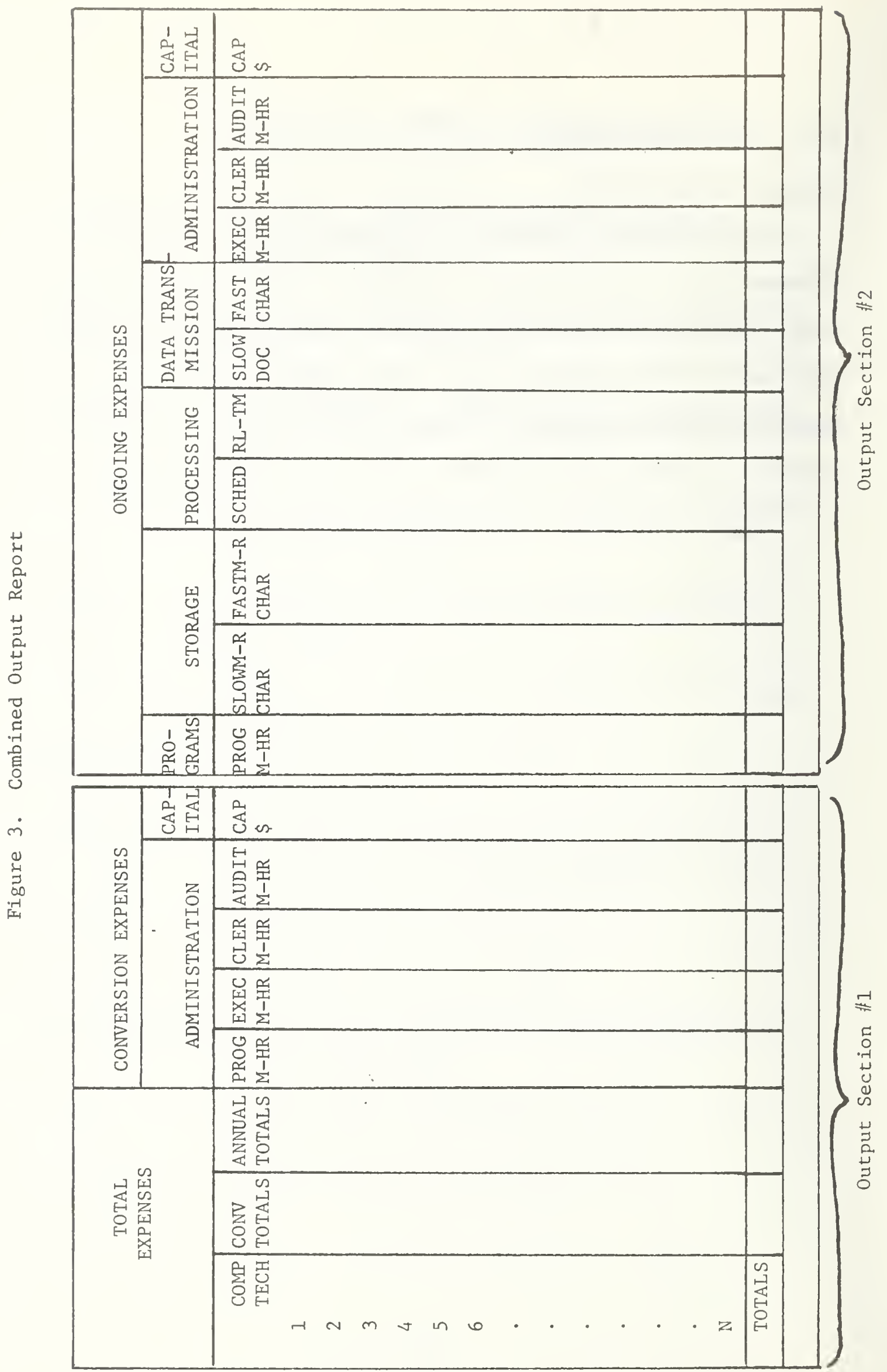




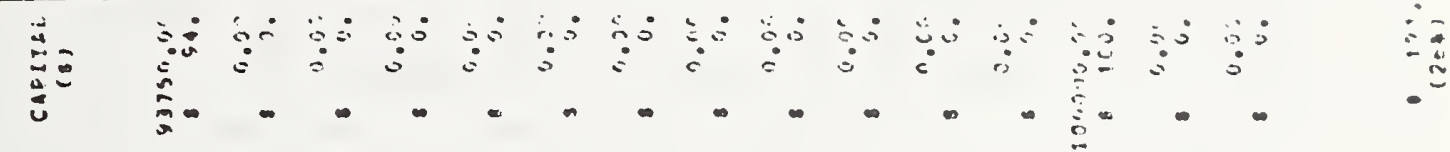

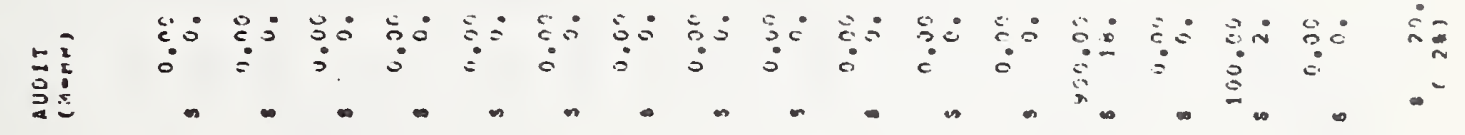

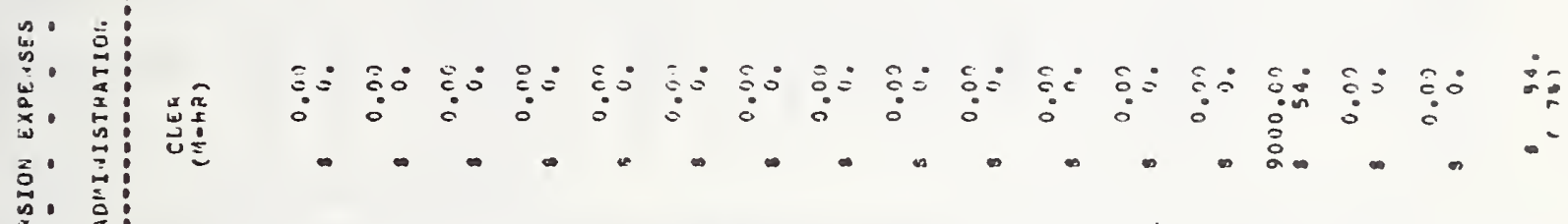

总, 言:

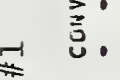

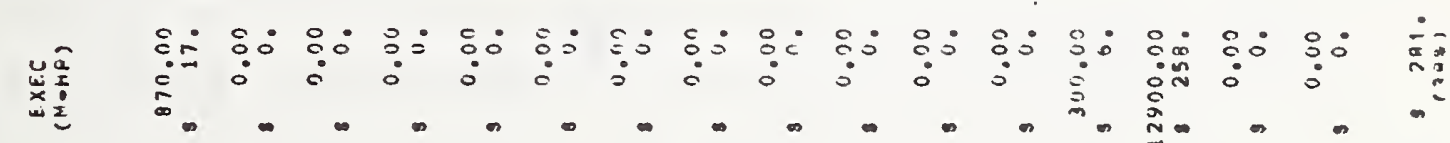

范

wr

音

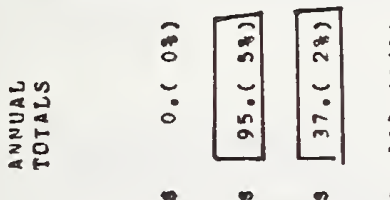

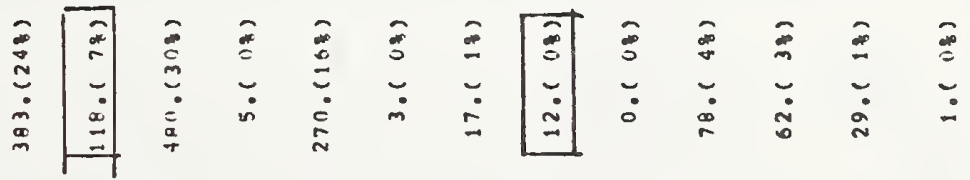

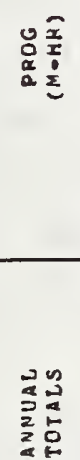

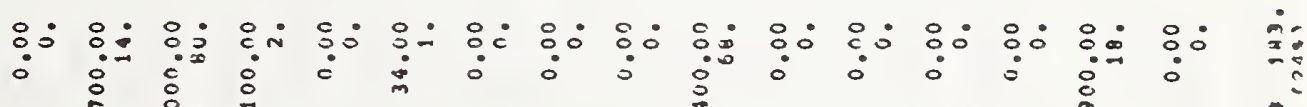

j

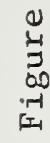

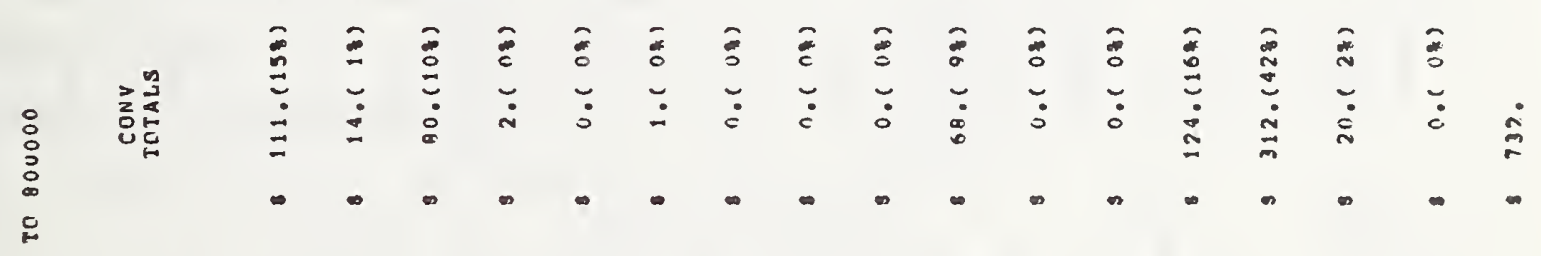

$\stackrel{5}{3}$

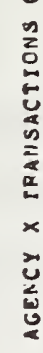

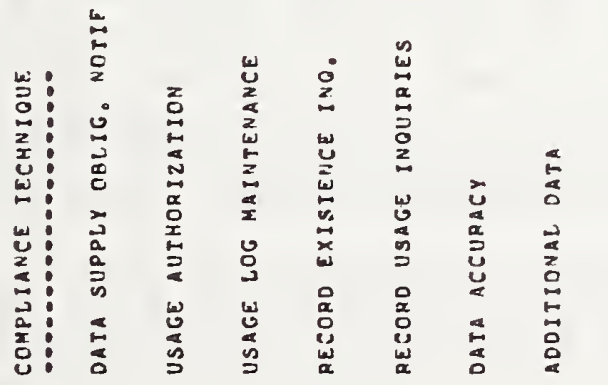

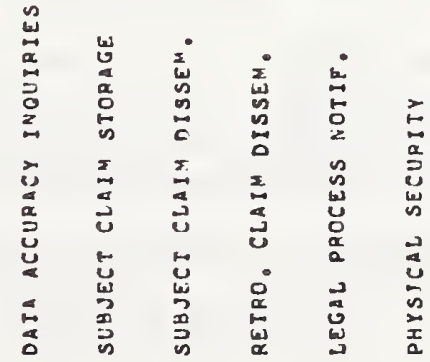

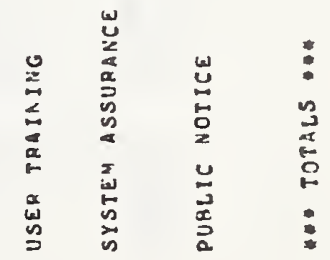




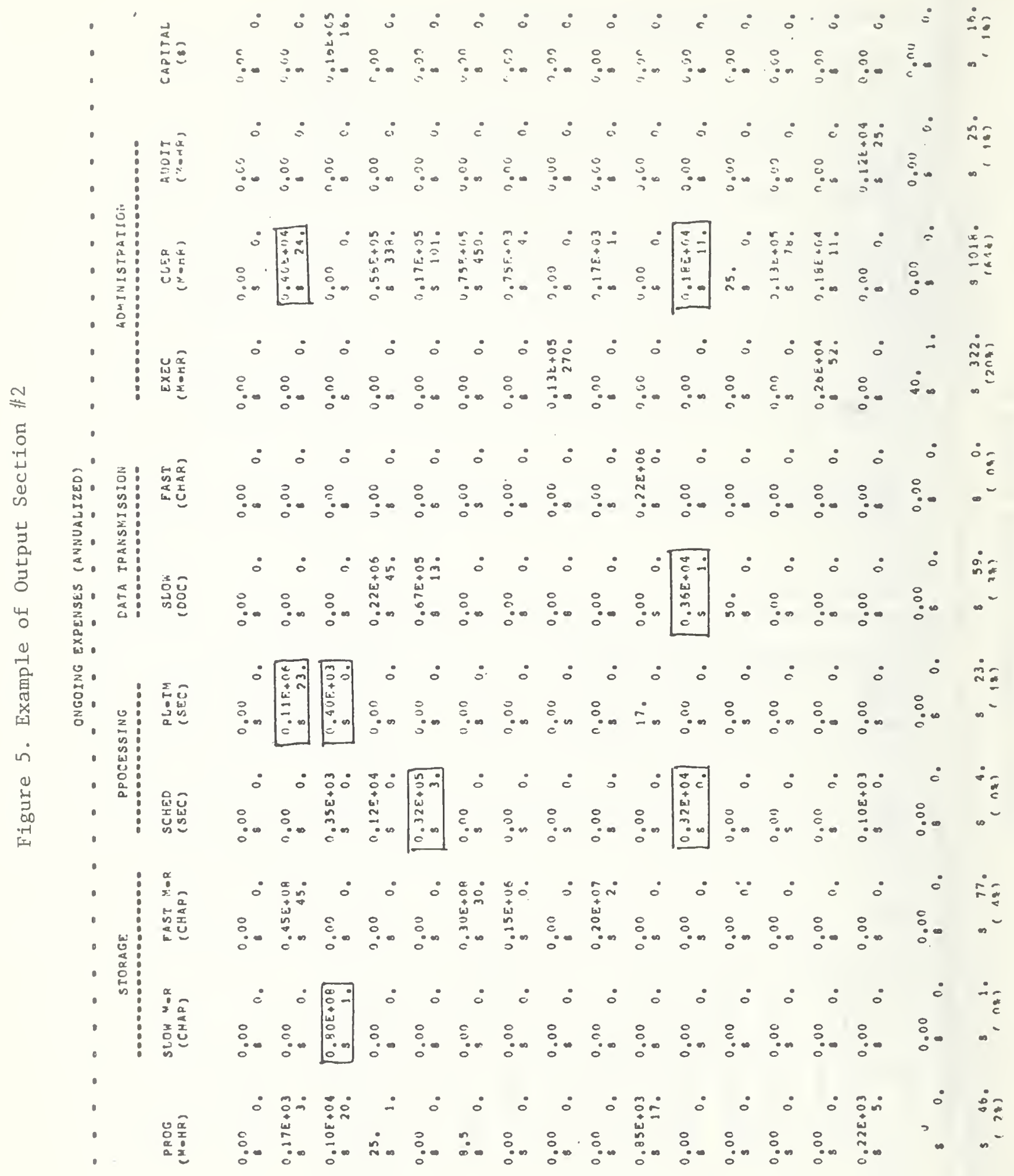

$-12-$ 


\subsubsection{Headers}

At the top of the "combined output" report, each of the required resources necessary to be expended in complying with the Privacy Act is printed. They are divided into six basic categories: Programming, Storage, Processing, Data Transmission, Administration, and Capital. Some of these (Storage, for example) are further divided into subcategories. In total, there are five columns for the conversion expenses and eleven for the ongoing expenses. Below each of the category headings is printed the units which are used in calculating that resource costs.

\subsubsection{Conversion Expenses}

Looking at the "Conversion Expenses" portion of the output, it should be apparent that there are two lines for each compliance technique (or step). The top line represents the quantity of resource units expended, and the bottom line represents the cost for those units. The user should note these costs are rounded to thousands of dollars. At the bottom of each column of resource, the costs have been totalled. The percentage of this cost to the total conversion expense is also shown in parenthesis below the cost. No calculation of total units required is made.

\subsubsection{Ongoing Expenses}

As with the conversion expense portion of the output, the ongoing expenses portion contains two lines for each requirement. The top line represents the quantity of resource units printed with an " $E$ ". They are decimal exponents and should be read as follows:

$$
\begin{aligned}
& .15 \mathrm{E}+03=.15 \times 10^{3}=150 \text { or } \\
& .27 \mathrm{E}+08=.27 \times 10^{8}=27,000,000 .
\end{aligned}
$$

The bottom line represents the cost for the units. These costs are rounded to thousands of dollars. At the bottom of each column of resources the costs have been summed. The percentage that this cost represents of the total annualized ongoing expenses is shown in parenthesis. No calculation of total units required is made. 


\subsubsection{Left-Hand Totals}

The left hand portion of the output report contains the total conversion and annual ongoing expense for each compliance technique. "Conversion Totals," for example, is the total of the five columns of conversion expenses located to the right. These numbers are printed in thousands of dollars. The conversion and annual expenses are summed vertically to obtain total conversion costs. Each compliance technique cost is then compared to the two totals and a percentage of total cost is shown. These are printed in parenthesis to the right of the cost value.

\subsubsection{Summary Output Report}

Obtaining the summary report is useful when the program is used in a time sharing mode and output is printed at the operator's terminal. It fits the 72 column teletype requirement quite well. It is also useful when performing sensitivity analysis since the analyst can see the effect of data changes quickly without waiting for a complete batch output.

As previously explained (see page 5g, Record 非1 Input Format), to obtain the summary report, a switch must be placed in the program to allow the summary columns to be printed without the detail.

The summary output contains the conversion and annual costs associated with each of the compliance techniques. These costs are described in thousands of dollars. The conversion and annual expenses are summed to give total costs for each. The percentage which each compliance technique contributes to the total cost is printed in parentheses to the right of each cost element. A sample Output Report is shown in Figure 6. 
Figure 6 - Summary Output Report

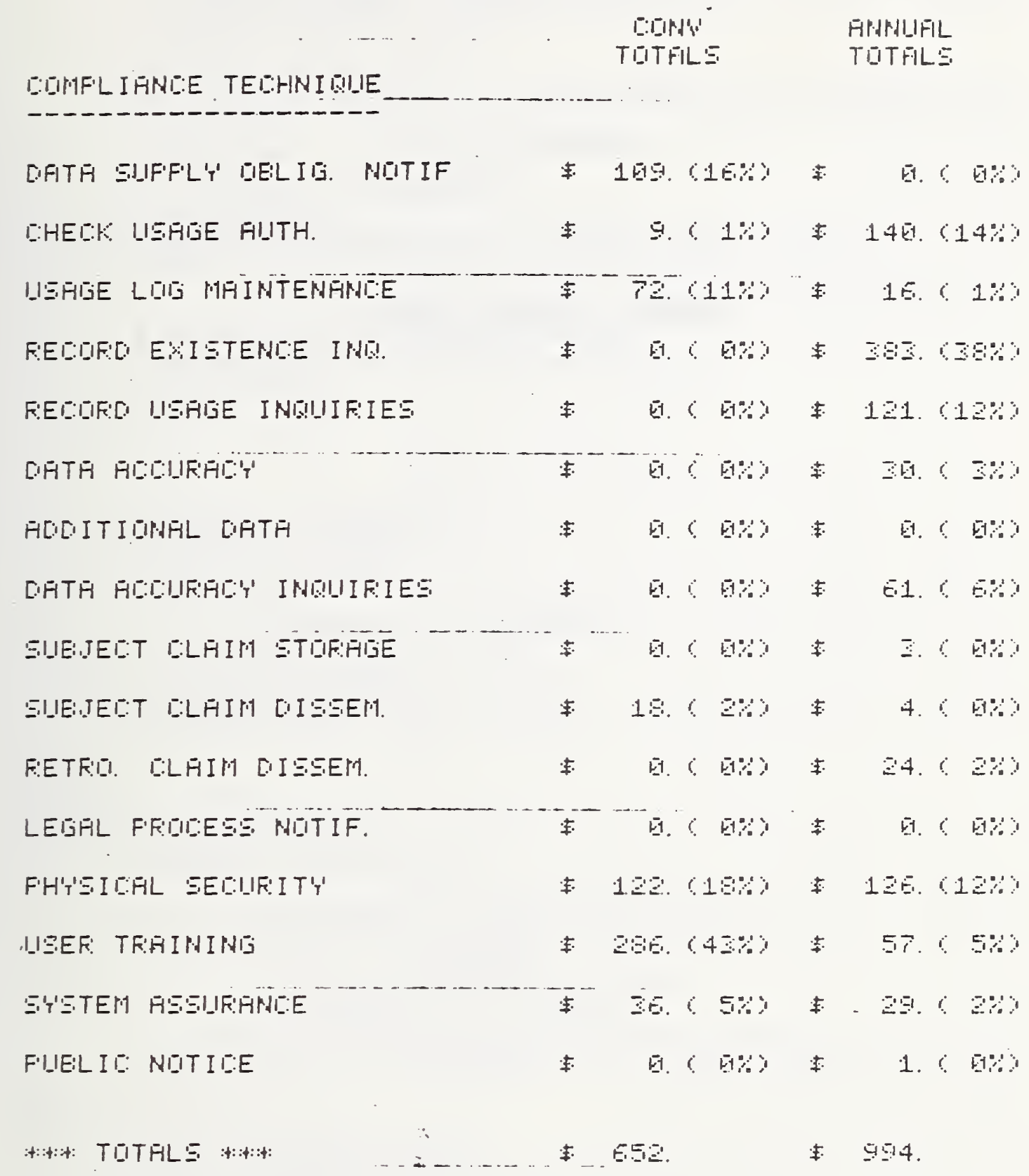

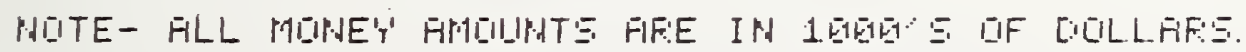


Appendix 1

COMPUTER PROGRAM LISTING

$-16-$ 


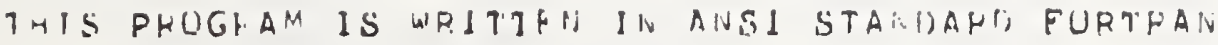

LT IMDIF MFMTS A MUDFI FUR KSTIMATJTG THF IMPACT UF lilf 197 A FLEIKAI, DRIVACY AC: IJ!I A

FIIRSONAL, MAIA SYSTEM.

THF ALGURITHMS USF, IN THE MERIFI, ARF. DESCRIAED IN DETA IL, IN

"THF COST UF PRIVACY"

HY RMRERT C. GOLDSTFIN

E'IIFI, TSHFI BY

HONEYHELL INEOPMATION SYSTEMS, INC, (1975)

A.j()

A METHIIUMINGI FUR EVALUATING

AITFRNATIVF APPPIACHF.S TO PRIVACY

KEOUIFFHENTS

HY

I:IMEFT C. GOMRSTHIH

IICHARD I IVOLAN

HEPIFY H. SE, WARI)

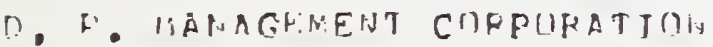

1 MIITIA LRIVE

I.F XINGIUN, MASSACHUSFTTS

(i) $17-8+2-4 \sin ^{11}$

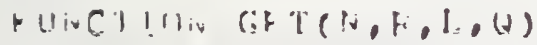

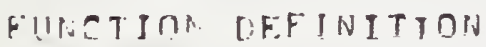

TTHF TU HFTHIEVE: A HECORI FRUM A FILE

H - "J" TE UNGINE;

W - NH: OF RtCOIRUS IN FIIE

L. i ingigh tif a RECGURD

Q PC. CIF RECLFIIS TII RF: HETHIEVEN

Dif 411

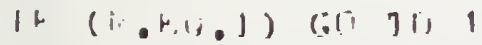

GeT = " D 10.001004167

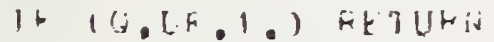

$G C .1=B * T_{1} * 0.00000333 / 0$

mbli'k

1 Get $=11.140$

rFIIII I

[ᄃ,

THF F[IIIIW]NG, ARRAY STZES AFE FOK 25 REOUIRFMENTS AND

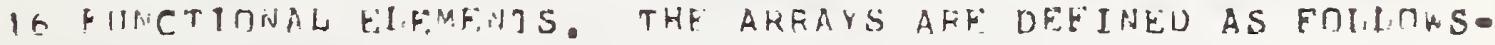

IJAN GURDTITY OF JTH FE WEEDED FIR ITH REOT

CIIEI CIST JE JTH FF IIEERET FUR IIH REET

UEST UNIT COST UF JTHFE

FECST TITAL COST FITH JTH PFE

F.CSTC CORVERSION COST FUR ITH RFOT 
1.GSIA AVP IIAL CIIST FIIP ITH PRAT

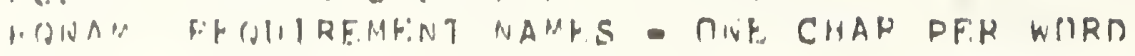

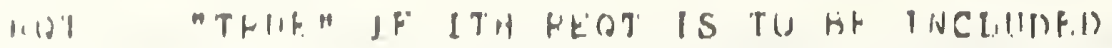

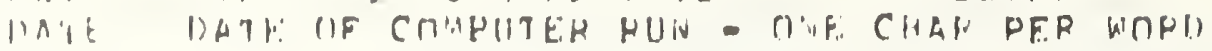

IITLF HUN JUFHTIFICATION - UN III RO CHAKACTERS

EF.AI, OUAP(23,16), COST $(25,16), \| C S T(16), F E C S T(16), \operatorname{RCSTC}(25)$

hEA I. h(.STh (25)

(1:1FGHR HHCTC(25), RFCTA (25), FEPCT(16), DNLN

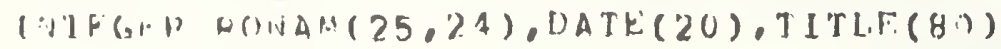

lí)G]CAL H.(i) (25)

Hit A1. WFRN, NSUH, ISCN, IFRH, L, NFFC, IWAC, INUS, INDRF, RRPR, NRLC, NUST, KLOG KF.AI. T RIN! ICACL, NRFI , NRIII, NDA I, NUTS, NADY, NLGS, NRTR, NLEN, KSEC RFA! ULSI, "CCI, TIHI, NCHK, HNSH, IWUL, NCPU

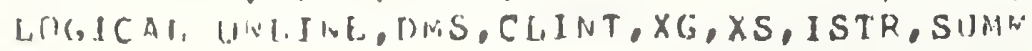

DAMANEIEP ASSIGNMENTS

MRQT NO. OF RFG TS IN THE MUDEL

IUFF NO. OF FE'S IN THE MLIDFL

INHIC AVG. NO. IF ACCFSS CUHTROL ENTRIFS PER RECMRD

WLiG MOP. OF 'TJHES PER YEAR USAGR LOG IS SEARCHE.D

FAOY FRACTION OF KECORDS IU NHICH "ADDITIONAL DATAM IS GDUEO (PF.K YEAR)

SCI.C SJZH IIF IISFR/OPEHATIR TRAJNING CLASSES

FTH ANMIIAL, THAINING VOLUME IS FRACTION OE IIITIAL

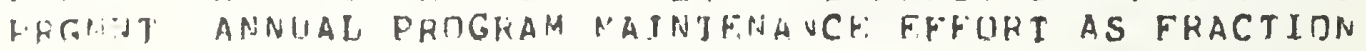
CIF INITIAL, DFVR.LOPMERT EFFUR

J DIIN FIIN COUNTFE

ISCV SCAN A FIIE (PEP RECOPI))

IFKM FOPMAT A SIMPISE RFOUIEST TO A DATA SUBJECT

ICICI, CHECK AH ACCESS COHIHII, IIST

IVAC WFITE AN ACCESS CONTKOL EHIFY

1)AIA UOT, NFE, WFLC, NLGS,FAUY, SCLS, FTR, PKC;MIT, IRUH

$1 \quad 120.16,0.5,52.0 .0 .0001,10.0,0.2 .0 .2 .01$

DATA ISC:, TFRH, ICACL,TWAC

$1 \quad 120010.1000 .1000 .500 .1$

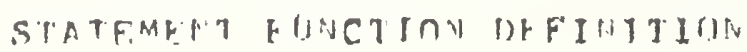

THE TO SORT R KECURUS UF LE:UR'T! I,

$\operatorname{sint}(1 ., 1)=R * L * 0.0(10) 21 R$

HIFIT HURMATS

8(101 FIJPA:AT(DGIO.0)

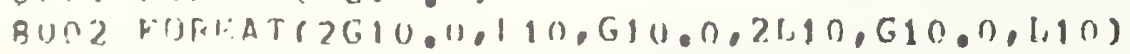

ROOB PIHAAT $(4610,0, L 10,610.0,21,10)$

80O. F FOKM $1(2511)$

BOOS FURA AT (ROAT) 


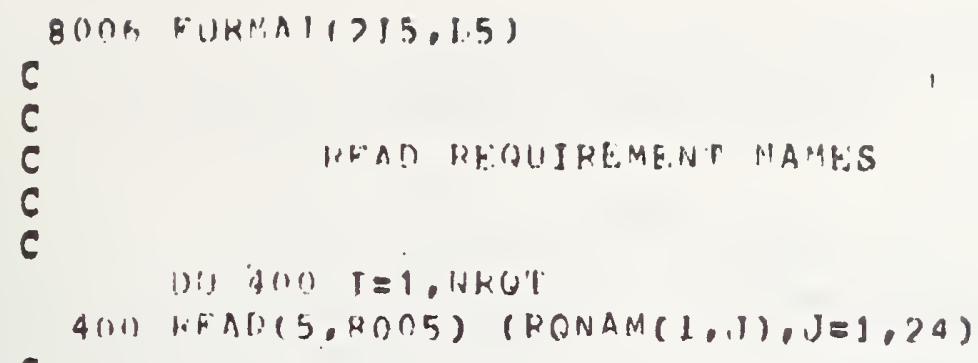

$c$
$C$
$c$
$c$
$c$
$c$
$c$
$c$
$c$

1:F.AI FUII DESCRIPTUKS

HHIH:S - NC, OR RIHS (SKITS UF JMPUT)

NCUIS - NO, QF OUTPII] CRIPIF.S TII HE PRINTED

SIIMA: - "JPUF" IF UNLY SUIAMAPY UITPUT

IS TU BE PRIITEO

KFAR (5,POCH) MEIIMS, NCOUS, SUMPS

BOO KFIAD) $(5, F \cup 05)$ (DATF, $(K), K=1,20)$

FFAI' $(5, F 005)$ (TITIE(K),K=1,80)

HEAI) (5,ROU4) (ROT (1),I=1, NKOT)

C

SYSTPM ATTRIBUTES

RFAU(5, HU1) I) NSUR, NREC, THRT, TVAL, NCHK, SHEC, NUSR

REAL $(5, H(1) \geqslant)$ NUST, NCLK, CLINT, NTFN, DNLINE, ISTR, NRPR, DMS

QEAD $(5,6 \cap \cap 3)$ SANIS, NFR', QFRM! NLPN, ISRC, TCEII, XG,XS

C

FF. COSTS

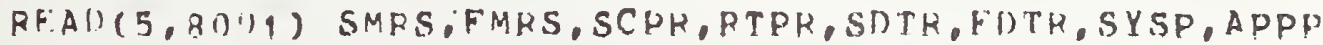
READ (5,E(U) 1) FXFC,CLEK, AlIDT

C

\section{STTES}

RFA! (5, MOOI) SADN, SCLN, SLOG, INUI, IINT, TANT

C

$M A N \cdot H \cap I P S$

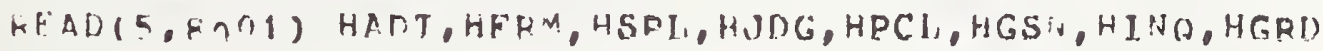

PFAI) (5, QR.11) HUJU,HCAD, HVEK,HTHC, HTRU

C

BFIRGAMNIIIG

RF:A[Y (5, \&OOI) HADJ, PUID, PNOT, PACC, PCVD, PUCL, FLOG, PRET MFAO(h,FIUI) FJAT,PWAC.

PITSET.LANFUUS

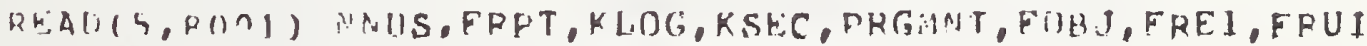
KED $)(5,2 R,(1))$ HDAJ,FRDS

SE T UN FARAIARTERS

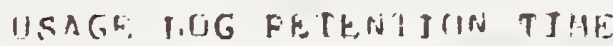

$11, G R=T K P T$

IF (TIGH.LT.5,) TLGP = 5.

$c$
$c$
$c$

SIZE OF SIIRJECT'S CIAIM

IF (SICLN.LT.6OO) SCLM $=600$. 
() 10 LI: $=11$

If (11,L I H. Fi) ININEI

$c$
$C$
$C$
$C$
$C$
$C$
$C$
$C$

C

C

C

C

SIZE OF USAGE IUG E゙NTRY

JE (SLOG.1.1.20.) SLIIG=20.

FRACIIUN OF EXXPIRING RECDHDS CHECKFI) PER YEAR

FVFH = NCHK/(NREC/TVAI,)

1:18HIFIHS AHII CUMPLAJNTS

WPEII = FREI NREC

$1 . F I I I=F F U I * N A E I$

WDAI $=$ WUAI NREI

WIUS $=(1 .-F F D S) * N D A I$

$c$
$c$
$c$
$c$
$c$
$c$
$c$
$c$

ADNITIONAL DATA TMCTIENTS

NADY $=F A D Y * N K E C$

CLFIH ACCUMLILATURS

(1) $2(: 1) \quad I=1$, inR(ST)

HCSIC $(1)=$ "

r. $\left.\operatorname{Cst} A_{0}(]\right)=11$.

1) $70 \div \quad 1=1.4 \mathrm{FE}$

200 $6(1 A,(1), 1))=i$.

(1) (1 2) $1,1=1, " F F$

$201 f+C^{\prime}(1)=0$

TCSTr: =

rCsin $=11$.

C

C

C

START UF IMPAC'T HOLF.L

1) ATA SUPPIY TBLIC, NOTIE, (F.) (3)

C

1 If 1 . A. IN . F(गT(1)) Gn TO 2

If $(A G)$ GO TU?

W

(J)A $(1,5)=$ A.FFMUFRM 0.015

$c$
$C$
$c$

CONSFHT HOF $A D D$ L USE (B)

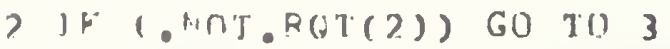

W!AN $(2,1)=$ PNUT + PNAC

GIAA $(2,6)=\operatorname{PRGMNT}$ UIIAN $(2,1)$

JUAR $(2,9)=M S W B *((1,+F R P T) *(T C P U *(I S C N+I F R A))+F \cap B J *$ 
1

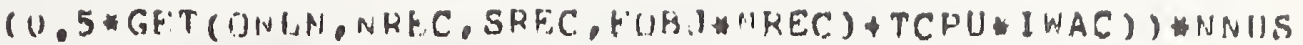
()$\left.^{i} / 4 \cdot 12,11\right)=N S I H *\left(1,+F+R+F\left(U H^{3}, 1\right)\right.$

\section{IISAGF: AUTHUPIZATION (H)}

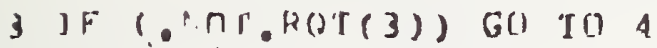

I. (1PH $=1 .+1.1 \%(N R P R-1$.

If (UMS) NTRP $=1$.

$(11 A:(3,1)=$ FUIR PACC*NI)RE

HUAM $(3, h)=\operatorname{PHGMNTUUUAN}(3,1)$

$S A C I F=12 . *(N U S T+N R E C * N K L C)$

IF (TIS,THE) (IUAN 3,8$)=S A C T K$

If (. "IT.ONLINE) UUAN 3,7$)=5 A C T K$

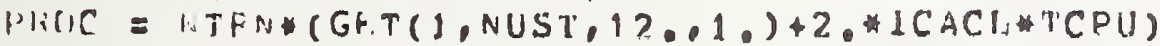

It $\left(U H^{\prime}, 1 \mathrm{NH}\right) \mathrm{OUAN}(3,10)=\mathrm{KRIC}$

IF $($, WTT.OHLINF) (JI)AN 3,9$)=P R O C$

IF (CI,IMT) (JUAN $(3,14)=$ THN*HIIN

USAGE LOG MAINTENALCE: (C) (1)

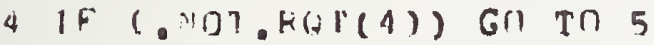

(UUAN(4., ) = PI.OG

UUA! $(4,6)=$ PF(GNATHRUAN $(4,1)$

WUAN $(7,7)=$ SLUG*NTAN*TLGR

PHIS = ITPN*ICPI 1 mUI,

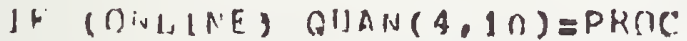

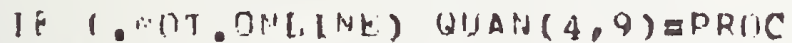

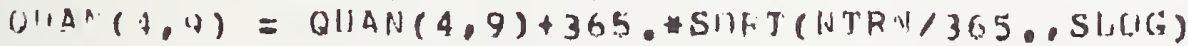

(vi)A. 14,16$)=K$ L.OG

- HFCRRD EXTSTFNCF \#DTJF.

(THIS KFOT WOT LNCLIIDED IN P.L.93-574)

S HOT $(5)=$.FALSE.

(j) 1110

C

PFCOHO EXISTENCF, WUUIRIES $(0)(1)$

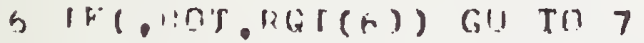

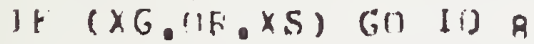

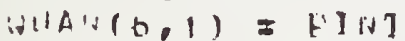

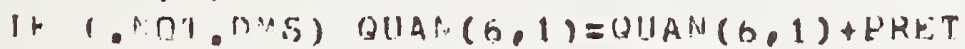

$($ W $A \cap(C, 0)=$ PRGM N I OUAN $(6,1)$

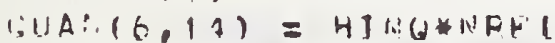

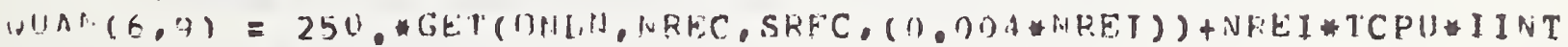
Uil $A r(n, 11)=A+F J$

I?HCOLA ISAGH INUHIRIES (C.) (3)

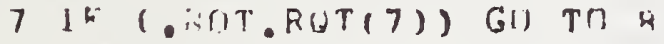

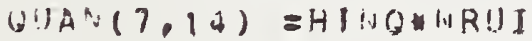

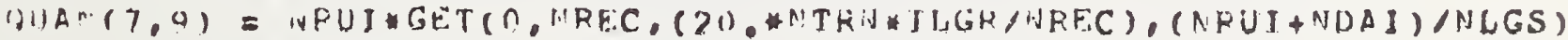

(1)A: $(7,11)=$ A.RU1 
hit (. 1,

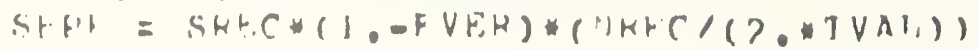

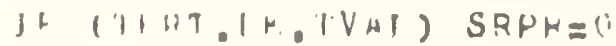

SIIR $=$ A. HAFC - SHHR

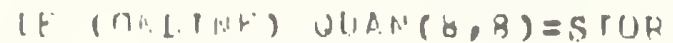

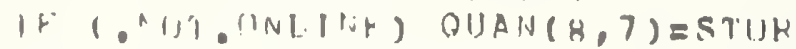

(NI) $\Delta 1 .(E, T)=F(V N)(1,+U .1 *(N K P H-1))$

(WIA $(y, \theta)=$ PH(

(.1)FA. (H., I ) = HVE.F*FVER*O.5*MREC/TVAL

\section{A1)1 IIIUHAI, DATA (F)(E)}

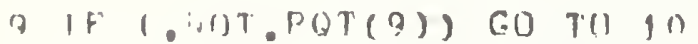

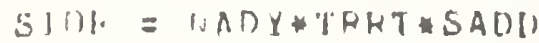

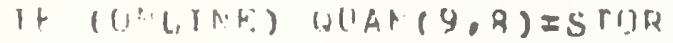

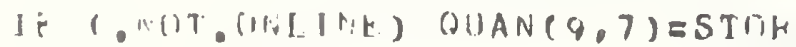

$1: H A:(9.1 .1)=$ HARY HCAO

\section{LEATA ACCUAACY IISUIRIES (N)(2)}

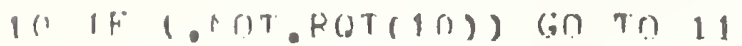

IH $(A G, \Gamma K, x S)$ GN 1014

: $(14: 110,13)=$ HCAI*HJDG

\section{SIMJFCT CLAJM STIRAGF (1))(3)}

11 IF 1.H. HCT(11) Gח TO 1 ?

GIHA $(11.14)=$ HF CL*UISS

SIMP = ! URS*TRHT SCIM

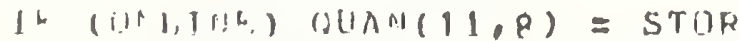

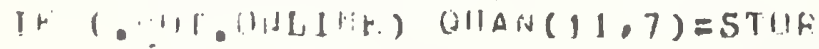

\section{SIIBJFC7 CLAIM DISSFAINASION (1)) (4)}

$1 \% 1 \div 1 . \because(1) .4(1)(12))$ G() Tn 13

(jiAf. $(12,1)=H$ U)CL*(1.+0.1*(NPR-1.) )

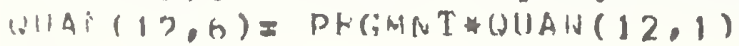

r.PS = 3110

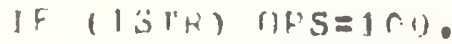

THE = IIFSHSAAS TCPU

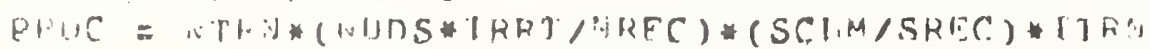

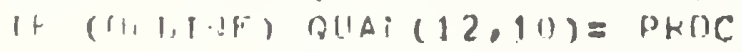

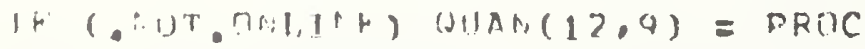

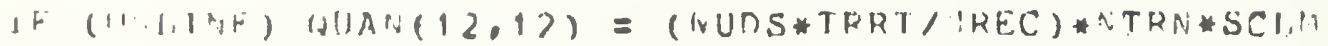

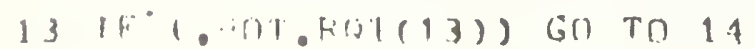

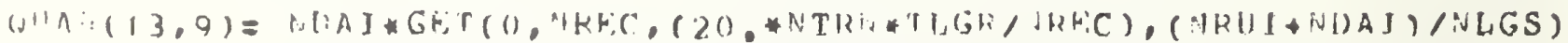

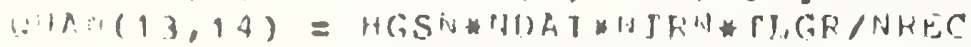

WHA. $(13,11)=$ WAI INTRN-TLGR/NREC 


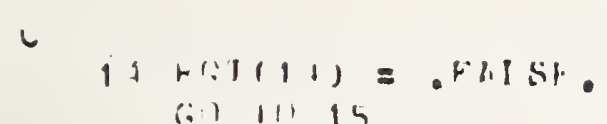

$c$
$c$
$c$

CMMSFN IO JPANSFFK [DATA (H)

$15 \mathrm{FiO}(15)=$.FALSF.

(i) il $1 \dot{n}$

$c$
C
C

LFGAI, ERLCESS NUTIHICATION (H)(Z)

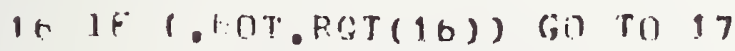

If $(x(;) \quad$ C. $] 1 ! 17$

$(1) \wedge i,(10,14)=A, P N \cup H G S N$

, $1.1,(1:, 11)=$ iv1 pis

C
c
C

\section{RHYSICAI. SECURITY (E) (10)}

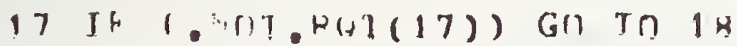

(:HA1.(17.2) $=0.25$ HSPL

(j:1A:(17,4) =0.75* HSPL

$(110:(17,5)=$ SEC

(1) $A 1,17,14)=$ HGRD

$c$
$c$
$c$

USEF TPAIHII: G (E)(G)

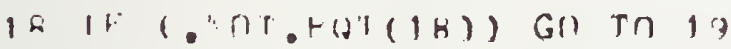

$1, H^{\circ}(1 \times, 3)=1 . C I, K * H 1 R C$

WIA: $(1 E, 7)=($ WI,K HTRC+1, HSK*HTRU)/SCHS

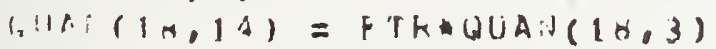

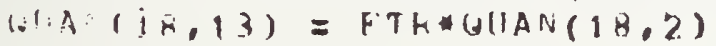

$c$
$c$
$c$

SYSTH.M ASSUHARCF

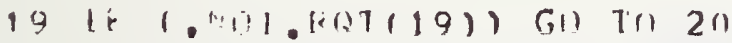

(31A $(17,1)=11.9 \%$ PHDT

$1,1 \cdot(19,1)=0.1 *$ PADT

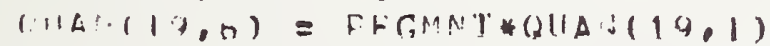

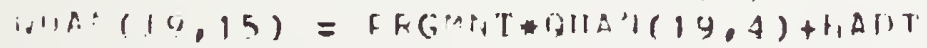

$(: 110 \cdot 1.10,0)=1 A !: T$

C
C
C

HIII IC. VIT ICE (F) ( +

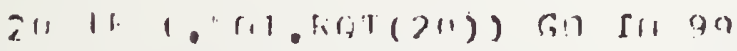

$\left(111 A^{\prime}(, i, 13)=10\right.$.

$c$
$C$
$C$
$C$

9) C111:11\%, 11: 
1,ESI, 1) = SYSH

Histis $; 1=1 \cdot x+c$

UCsir 3$)=\operatorname{Clith}$

$\operatorname{licsi} 4$ ) $=\Delta$ IInT

(ICS), b) $=1.0$

('CST $(6)=$ SYSP'

VCSI ( 7) = SMFS

UEST (R) = PMHS

$\| C S I(9)=S C P R$

UCST $(10)=$ RTPF

JCSI $(11)=$ SNTH

(ICSI 12$)=$ FIITK

(ICST 13$)=E \times F C$

$U C S)(14)=C L, F$

11C5T(15) = Al117

$1 ! \cos 1(16)=1.0$

$0 ! 1 \quad 131 \quad 1=1,1, R(y)$

It $\left(.1,1 . D_{(g I}(\mathrm{I})\right) \mathrm{G} U \mathrm{TO} 111$

nIC 1 (N).$=1$, NFE

$\operatorname{Cos} 1(1,0)=\operatorname{UtAN}(1, J) * \operatorname{LCST}(J) / 1000$.

FECST $(1)=\operatorname{FrCST}(3)+\operatorname{Cos} 1(1,0)$

C SHFAFATH COUVERSION FROM AUHUAL CHSIS

1. (J.61.5) Gri T(i 1111

C CIMVERSIUH! COSTS

$\operatorname{lisic}(1)=F \operatorname{cst}(1)+\cos (1,1)$

$\operatorname{cosic}=\cos 1 \mathrm{C}+\cos (\mathrm{cos}, \mathrm{J})$

(B) 11,1611

C

Ai:HUAL COSTS

101 HCSIATI $=\operatorname{HCSTA}(1)+\operatorname{CIIST}(1,1)$

ICSTA $=1 \operatorname{csta}+\cos (1,1, J)$

$\ln [10[0,1 \%]) \cdot 1014$

111 CHADII

C

C

$c$

C

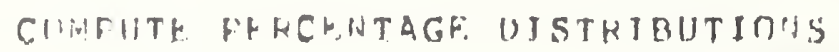

I:!) $30(1) \quad 1=1, \mathrm{NR}(\mathrm{JT}$

riCl( 1$)=100$ RCSTC(J)/TCSTC

$31)+H C(A(1)=100 . \#$ FCSTA(1)/TCSTA

Ciा $3(1), 1=1, N F F$

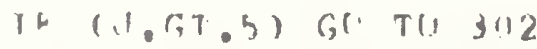

FHCl( 1$)=100 *$ FHCST (.1)/TCSTC

$6 !: 11 ! 31: 1$

$36 \%+F(1)=100.4 \operatorname{FCST}(1) / \operatorname{TCSTA}$

3131 C.TH 1 1.

C

C

C

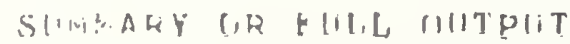

C

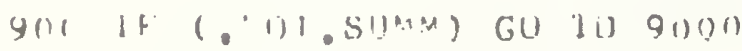

C

C

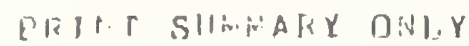

c

MR 1$]+(h, 9101)$ (TJ TLE $(K), K=1,6(i),($ DATE $(K), K=1,20)$

91119 FIIRAAT ( IHI, 1OX, 19HJ

$111 x, 004 / 11$, 20A1////45X, AHCORV, 8X, GHANAUAJ,/

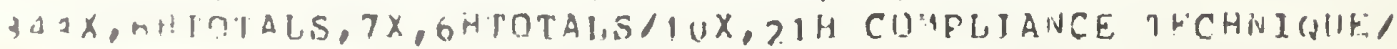


$.11 \times, 2:(1,-1)$

$c$
$C$
$c$
$c$

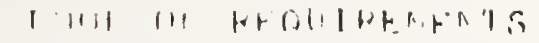

$111,111,1=1, \therefore$ fint

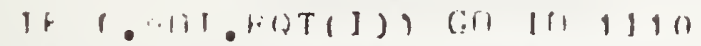

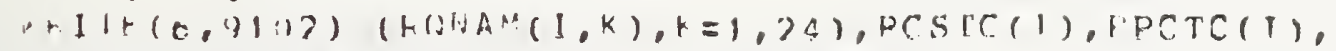

ihEsiA (1), FDCTA(T)

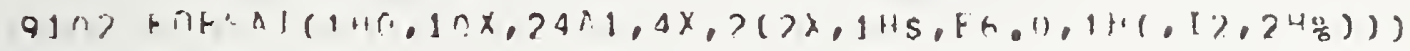

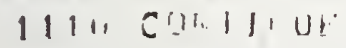

c

C

C

C

C!I 11? THTALS

$-11+1 m, 011,1)$ TCSIC, TCSTA

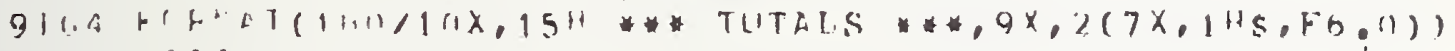

i?] $11,12, g(0,15)$

s.t $(1+(t, y)$, 105$)$

(3) is timitar,

(:!) $111 \quad 19011$

$c$
$c$
$c$
$c$

["I I I I.LF HALF FIHST HKADJACSS

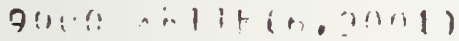

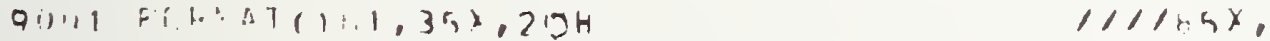

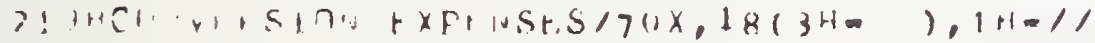

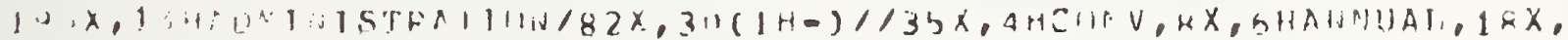
$?$ H

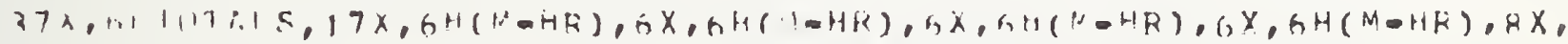

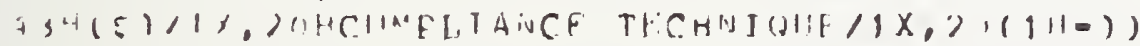

$c$
$c$
$c$
$c$
$c$

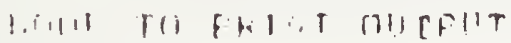

(i) $11: 1=1, r$, 1,1

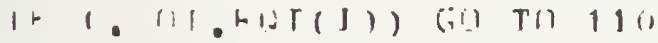

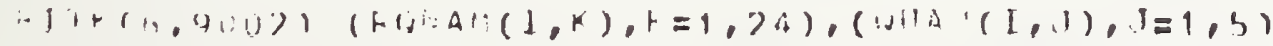

$9(1) \cdot 11 ; 1(1,1,2) 81,411,5(3 x, F 4,2))$

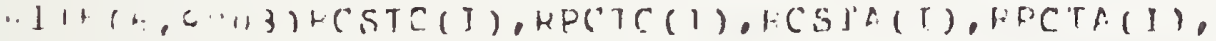

$1(\{1,511,1), 1=1,5)$

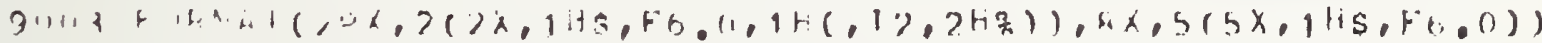

$11 \cdots 6+1,1\} 1,1$

$c$
$C$
$c$

\section{G I.1 ?: $107 \% 1,5$}

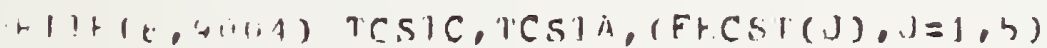

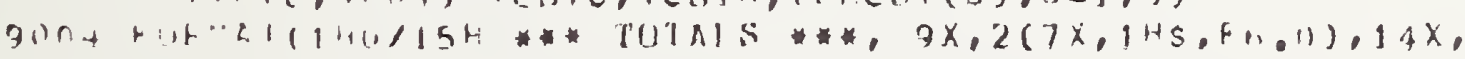

$15,(5 x, 1+5,(0,0))$

$\therefore(1,10,0,14)$ (FECT $(19), 1=1,5)$

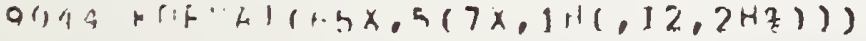

(.) $11+(n, 0)(1,15)$

91)1:5+1. $11(1+1 / 11) x$,

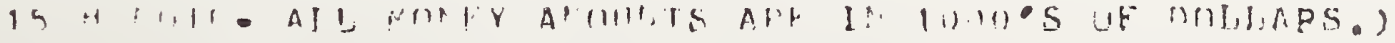




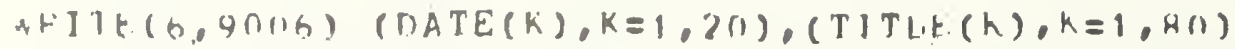

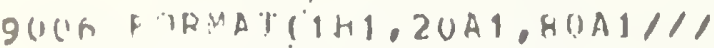

$11 H(1,52 X, 29 H O N G U 1]$ E EXPFNSES (ANIUALIZEV) $/ 4 X, 42(3 H-$ ), $1 H-1 /$

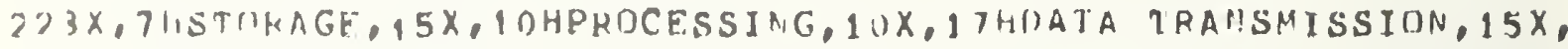

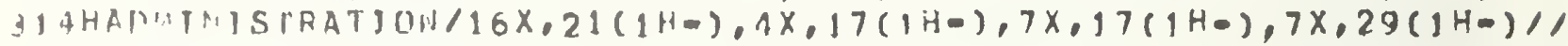
$45 X, 4 H P I I G, 7 X, 8 H S L \Pi N M=K, 5 X$, BHFAST $M-R, 4 X, S H S C H E D, 7 X, 5 H R I-T M, B X$,

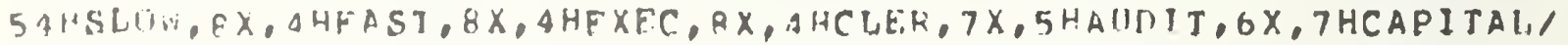
$64 \dot{6}, 5 H(\cdots H K), 7 X, 6 H(C H A R), 6 X, 6 H(C H A R), 6 x, 5 H(S E C), 7 X, 5 H(S E C), 7 X$, $c$
$C$
$C$
$C$ $75+([H, C), 7 \times, 6 H(C H A H), 3(6 X, 6 H(M-H F)), 7 X, 3 H(S) / /)$

\section{LOUP in FHIT nUTPUT}

r.11 $1701=1, \mathrm{NR}(\mathrm{NT}$

If $(.0 T$. F(US (1)) GU 10120

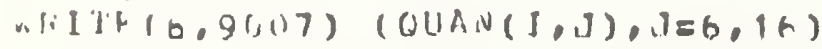

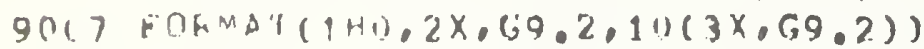

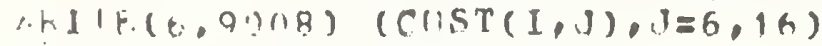

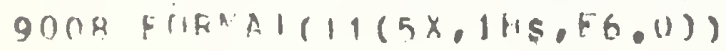

$12(1$ Clin: $111,1,4$

C
C
C
C

CIIILI:4N TGIALS

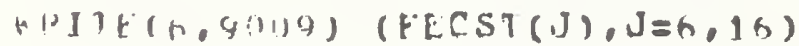

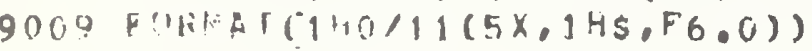

NAl I R. (n, 71109 ) (FFPCT $(J), J=6,1 \mathrm{~h}$ )

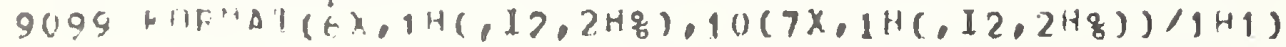

C

C

C

FAN UF REROHF = CHECK FOR MORE

$11211,1 C H P=1 C H P+1$

If (ICHP.1.N.NC(IFS) GI) TO 300

$3+10 \%=1$ inlinit 1

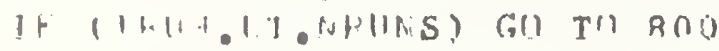
if ThISHER

STr,

F.'L: 
APPENDIX 2

GLOSSARY 
APPP - Cost of applications programming.

AUDT - Cost of auditor time.

CLAS - Characterization of system.

CLER - Cost of clerical time.

CLINT - If there is a clerical intermediary in obtaining personal data, CLINT $=\mathrm{T}$, if not CLINT $=\mathrm{F}$.

DMS - A logical variable DMS=1 if a DBMS exists, 0 if it does not. A DBMS negates the requirements for PRET.

EXEC - Executive costs.

FADY - The percentage of records to which additional data will be added each year.

FDAI - The percentage of individuals making a record existence inquiry who challenge something in their record.

FDTR - Cost of fast data transmission (Telecommunication).

FOBJ - The percentage of data subjects who may be expected to object to a proposed new use for their information. If a subject objects, his record must be so annotated and blocked from use.

FMRS - Cost of fast (Online) machine readable storage.

FRDS - Frequency of data accuracy disputes resolved in agency's favor.

FREI - The percentage of records in the system that will be subject to record existence inquiries per year.

FRPT - The percentage of NSUB which will require follow-up letters to obtain consent.

FRUI - Frequency of record usage inquiry.

FVER - The percentage of records expiring each year that are reverified.

GET - GET is a function used by several of the algorithms to determine the time necessary to get a record from the file. There are four items used by the GET function (N,R,L,Q).

$\mathrm{N}=\mathrm{T}$ is the data base is online. If this is true, GET $=.14$. This parameter may be varied when it is necessary. 
$N=0$ if offline

$R=$ Number of records in file

$\mathrm{L}=$ Length of a record

$Q=$ Number of records to be retrieved

If $Q$ is less than or equal to one, one half the file must be read and access equals

$$
\mathrm{GET}=\mathrm{R} * \mathrm{~L} * 1 / 120,000 / 2
$$

If $Q$ is greater than 1 and $N=0$, the entire file must be read and

$$
\mathrm{GET}=\mathrm{R} * \mathrm{~L} * 1 / 120,000 / \mathrm{Q}
$$

The GET function is based on the following assumptions about file organization and usage:

Online data bases are assumed to be organized using the Indexed Sequential or equivalent method. Access to a single record, at random, will then require, on average, four disc accesses. A time of 35 milliseconds per access is used as being reasonably typical of today's high performance disc systems.

Offline files are assumed to be stored on tape using technology that permits reading the tape at a rate of 120,000 characters per second. If only one record is to be retrieved, one half the file must be read, on the average. If more than one record is to be retrieved during a single search, it is assumed that the entire file will be read and the total time allocated equally among all the records retrieved. 1/120,000 is the amount of time to read one character in the file.

HADT - Manhours per year of audit time to assure compliance with the Privacy Act.

HCAD - Clerical hours to obtain and enter one item of additional data. HFRM - Manhours required to redesign a form.

HGRD - Manhours required to guard equipment, check identification, etc. HGSN - Clerical hours to process a legal response.

HINQ - Clerical hours required to process an inquiry and subsequent clerical tasks. 
HJDG - The number of hours required to receive and act on one data accuracy inquiry.

HPCL - The hours needed to prepare and enter one subject's claim.

HTRC - Number of class hours necessary to train clerical personnel.

HSPL - Hours required to develop a physical security plan and audit procedures.

HUID - The number of hours of clerical time required to confirm the identity of a person submitting an inquiry.

HTRC - Hours required to train operators in security and privacy.

HTRU - Hours required to train users other than agency personnel. It is assumed that the agency maintaining the data base will train other agency users.

HVER - Clerical hours to reverify one expiring record.

IFRM - The number of CPU instructions to format a request to data subjects.

IINT - Number of instructions to generate a comprehensive hardcopy or records.

ISCN - Instructions to read next record.

ISRC - Personal data from subject.

ISTR - A logical variable whose value is true if the system primarily processes structured inquiries and false if inquiries are primarily unstructured. Unstructured inquiries require greater amounts of transaction processing time.

IWAC - The number of CPU instructions to write control access information.

IWUL - Number of CPU instructions to write data in usage $\log$.

KLOG - The annual dollar cost of additional equipment needed to maintain the usage $\log$.

KSEC - The dollar value of additional equipment needed to achieve an appropriate level of security.

NADY - The number of additional data fields added to the system per year. 
NCHK - Number of existing ("Old") records checked each year.

NCLK - The number of clerical personnel associated with operation of the system.

NDAI - Number of data accuracy inquiries per year.

NDRP - The number of application programs which directly access the data base. $N D R P=1$ if a general data management system is used to handle all requests for data by applications programs.

NFRM - The number of distinct data collection forms used by the personal data system.

NLGS - The frequency of searching the usage log per year.

NLPN - The number of legally enforceable requests for data received per year.

NNSB - Number of new subjects added to file per year.

NNUS - Number of new uses for the data base each year.

NREC - Total number of data subject records. It may be greater than NSUB as one individual may have more than one record in the data base.

NREI - Number of record existence inquiries received per year.

$$
\text { NREI }=\text { FRE } I * N R E C
$$

NRLC - The number of record level access control fields per record.

NRPR - The number of record retrieval programs utilizing the data base.

NRUI - Number of record usage inquiries per year.

NSUB - The number of individuals about whom identifiable personal data is stored in the system.

NTRN - The average number of transactions processed by the system per year.

NUDS - The number of data accuracy inquiries that are not settled and must be added to the data base per year.

$$
\text { NUDS }=(1-F R D S) * N D A I
$$

NUSR - Number of potential users of the system. 
NUST - The number of sets into which all users can be lumped for access control purposes.

ONLINE - ONLN

ONLN - If the data base is online, ONLN=1. If the data base is offline, ONLN=0.

OPS - Variable which $=300$ if system inquiries are unstructured and 100 if inquiries are structured. Used with ISTR. Unstructured inquiries are assumed to require three times as much processing time as structured inquiries.

PACC - Programming hours to implement record level access control.

PADT - Programming hours needed to prepare all the programs required by system auditors.

PCVD - The amount of programming hours to implement the verification date check in one record retrieval program.

PDCL - The programming hours to modify a record retrieval program to include the claim field in its response to all inquiries.

0.1* (PDCL) - The additional hours necessary to modify subsequent retrieval programs after the first program has been modified.

PINT - Programming hours to produce a copy of all the information relating to a specified individual in comprehensible form.

PLOG - The amount of programming hours required to implement the usage log capability.

PNOT - The amount of programming hours necessary to generate a program for writing form letters to all data subjects.

PRET - Programming hours to permit retrieval of all the data on a specified individual if no data base management system (DBMS) is used.

PRGMNT - The percentage of initial programming hours required annually for program maintenance.

PRSB - Print subject's file in "comprehensible" form.

PUID - The programming hours required to add a user identification to the system.

PWAC - The amount of programming hours necessary to generate a program to permit the recording of all access control information within the data base and within each subject's record. 
QFRM - Inventory level of each form on hand.

QUAN $(i, j)$ - An array used for programming and convenience. The $i$ subscript relates to the compliance step. The $j$ subscript relates to the type of resource.

\section{i Values}

1. Data Supply Obligation Notification

2. Consent for Additional Use

3. Check Usage Authorization

4. Usage Log Maintenance

5. Record Existence Notification (not required by 1974 Privacy Act)

6. Record Existence Inquiries

7. Record Usage Inquiries

8. Data Accuracy

9. Additional Data

10. Data Accuracy Inquiries

11. Subject Claim Storage

12. Subject Claim Dissemination

13. Retroactive Claim Dissemination

14. Record Transmission (not required by 1974 Privacy Act)

15. Consent to Transfer Date (not required by 1974 Privacy Act)

16. Legal Process Notification

17. Physical Security

18. User Training

19. System Assurance

20. Public Notice

\section{j Values}

1. Programming (Conversion)

2. Executive Time (Conversion)

3. Clerical Time (Conversion)

4. Auditor Time (Conversion)

5. Capital Expenditure (Conversion)

6. Programming (Annua1)

7. Offline Machine Readable Storage (Annual)

8. Online Machine Readable Storage (Annua1)

9. Schedulable Computer Processing (Annual)

10. Rea1 Time, Online Computer Processing (Annual)

11. Slow Data Transmission (Annual)

12. Fast Data Transmission (Annual)

13. Executive Time (Annua1)

14. Clerical Time (Annual)

15. Auditor Time (Annual)

16. Capital Expenditure (Annual)

RTPR - Cost of instantanous processing. 
SADD - The amount of storage space in characters that would have to be allocated for each occurrence of an additional data field.

SANS - Size of average inquiry answer in characters.

SCLM - Characters needed to express one subject's claim.

SCPR - Cost of schedulable processing.

SDTR - Cost of slow data transmission (U.S. Mail).

SLOG - Size of $\log$ entry.

SMRS - Cost of slow (Batch) machine readable storage.

SYSP - Cost of systems programming.

TADT - The amount of computer hours required, per year, to support the system auditor.

TCPU - Time in seconds required to execute 1 instruction.

TLGR - Length of time an entry is maintained in the usage log in years.

TTRN - The computer time in seconds needed to process one transaction. TTRN $=$ OPS $*$ SANS*TCPU

TRRT - Number of years a record is retained.

TVAL - The number of years that information can be considered valid.

$X G$ - If agency is exempt due to law enforcement $X G=T$, if not, $X G=F$.

$\mathrm{XS}$ - If agency is exempt for other reason $\mathrm{XS}=\mathrm{T}$, if not, $\mathrm{XS}=\mathrm{F}$. 


\author{
Appendix 3 \\ Patterned Interview Format for Data Gathering
}

1. This appendix contains the questions used to obtain the data base input necessary to use the privacy model. The specific datum is listed below the question.

2. Questions without a specific datum are general in nature and are used to get general information concerning the data base. 
Data collection

Section I: System Attributes

1. Which of the following application areas best characterizes your system? (If more than one applies, please indicate the most important one, and answer all remaining questions with respect to just that part of your total system.)

.... Credit

.... Education

.... Employee (Not a program input)

..... Health

.... Insurance

.... Law Enforcement

..... Welfare

..... 0ther (Please specify:.... CLAS............

2. How many individuals are subjects of identifiable information in your system?

NSSỤB.

Number of individuals

3. How many separate records does the system contain? (Some systems may be organized with more than one record per individual.)

NRE.C.

Number of records

4. How many new individuals are added to the system in an average year?

NNSB. (Not a program input)

Number of individuals 
5. For how long are records retained within the system?

$$
\text { Number of Years }
$$

6. For how long (on the average) is data about an individual valid for its intended use?

$$
\text { . TVAL..... }
$$

\section{Number of Years}

7. Is there a regular program of periodically revalidating information?

$$
\text { Yes.or. No (Not a program input) }
$$

8. If so, how many "old" records are re-checked each year?

$$
\begin{aligned}
& \text {.. NCHK.... . } \\
& \text { Number of Records }
\end{aligned}
$$

9. What is the average size of a record (in characters)?

$$
\text { SREC }
$$

\section{Number of Characters}

10. How many individuals are potential users of your system; that is, how many may initiate transactions?

........

Number of Individuals

11. If these users can be lumped into sets for access authorization purposes, how many such sets are there?

$$
\text { Number of Sets }
$$


12. How many individuals are involved in the operation of your system including data entry and other clerical tasks?

$$
\begin{aligned}
& \text {...CLK ... } \\
& \text { Number of Individuals }
\end{aligned}
$$

13. Are inquiries made:

CLINTEF directly by the person wanting the information, or CLINTET by a clerical intermediary?

14. How illany transactions are processed per year (on the average)? ... NTRN....

Number Transactions/Year

15. Are transactions processed:

OULINE=T interactively, or

ONLINEET using online batch techniques, or

ONLINEEF offline?

16. Does your system permit:

IST TR=F only specific, highly structured queries, or ISTR:T unstructured browsing?

17. How many transaction processing programs exist in your system? . NRPR. . . .

Number of Programs 
18. Do transaction processing programs access the data base:

. DMS. directly, or

.... through a general purpose data management program?

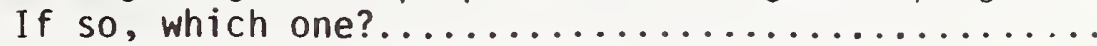

19. What is the average size (in characters) of the answer your system provides to a query?

SANS....

Characters

20. How many different data collection forms do you use?

. NFRM ....

Number of Forms

21. How many copies of each data collection form are normally on hand? . QFRM....

Number in Stock

22. How many times per year do you receive a legally enforceable request to supply data about an identifiable individual?

. NLP.N ....

23. Does most personal data in your system come from:

ISRC $=T$ the subject, himself, or

ISRC=F. someone else? 
24. What computer model(s) is (are) used to run this system?

$$
\begin{gathered}
\text { L.ist. Computsers. } \\
\text { (Not a Program Input) }
\end{gathered}
$$

25. Is your system accurately described by the following:

"a system of records maintained by an agency or component thereof which performs as its principal function any activity pertaining to the enforcement of criminal laws."

$$
\begin{array}{ll}
\ldots X G \ldots . . . & \\
\text { Yes or No } & \text { Yes }=T \\
& \text { No }=F
\end{array}
$$

26. Is your system accurately described by the following:

"a system of records--

(1) of investigatory inaterial compiled for law enforcement purposes (other than those described in the preceding question);

(2) maintained in connection with providing protecting services to the President;

(3) used solely as statistical records;

(4) compiled solely for the purpose of determining eligibility for Federal civilian employment, military service, Federal contracts, or access to classified information;

(5) of testing or examination material used solely to determine individual qualifications for appointment or promotion in the Federal service;

(6) evaluation material used to determine potential for promotion in the armed services.

$$
\begin{array}{ll}
\ldots . . X S \ldots . . . & \\
\text { Yes or No } & \text { Yes }=T \\
& \text { No }=F
\end{array}
$$


Section 1I: Resource Prices

This section asks you to estimate the unit costs tu your organization of various information processing resources. Please include in your estimates any overheads or other indirect costs that may be associated with ear.h i tell. DON'T FORGET TO STATE YOUR UNITS FOR EACH ANSWER. If costs are difficult to deterinine, can you supply numbers of people involved or other indicators of cost.

27. Sequential-access, machine-readable storage - i.e., magnetic tape or punched cards:

$$
\begin{aligned}
& \text {.. SMRSS... } \\
& \$ / \text { Character } / \mathrm{Yr} \text {. }
\end{aligned}
$$

28. Randon-access, machine-readable storage - i.e., magnetic disks or druilis, or bulk core:

FMRS...

$$
\$ / \text { Character/Yr. }
$$

29. Schedulable computer processing - that is, processing which may be preplanned for convenient times:

$$
\text { ...SCPR... }
$$

$\$ / \mathrm{Sec}$.

About what percentage of total computer processing is schedulable? (Not a Program Input)

30. Non-schedulable computer processing - that is, processing which inust be performed instantly when demanded:

$$
\begin{gathered}
\text { RTPR.... } \\
\text { \$/Sec. }
\end{gathered}
$$

Percentage of total processing non-schedulable:

(Not a Program Input) 
31. Slow data transmission - i.e., U. S. Mail:

$$
\begin{aligned}
& \text { S. STIR... } \\
& \text { \$/Doc. }
\end{aligned}
$$

32. Fast data transmission - via communications lines:

$$
\begin{gathered}
\text { FDTR } \\
\text { \$/Character }
\end{gathered}
$$

33. Systems programming - including related computer time and other support

$$
\begin{aligned}
& \text {..SYSP.... } \\
& \$ / \text { Hour }
\end{aligned}
$$

How many people?

(Not a Program Input)

34. Applications programming - including computer and other support:

$$
\text { ...PPP.... }
$$

How many people?

$$
\text { (Not a Program Input) }
$$

35. Administration - policy creation and review, training, enforcement of regulations:

$$
\begin{gathered}
\text { EXEC } \\
\text { \$/Hour } \\
\text { How many people? }
\end{gathered}
$$


36. Clerical processing - data entry, filing, handling inquiries: ... CLER...

$$
\$ / \text { Hour }
$$

How many people?

$$
\text { ....... (Not a Program Input) }
$$

37. Audit - system performance monitoring - quality control

$$
\begin{aligned}
& \text { AUDT .... } \\
& \text { \$/Hour }
\end{aligned}
$$

How many people?

(Not a Program Input) 
Section III: Sizes

These questions refer to the amount of computer storage space that would be required to hold various items of information.

38. The size of the field that would be required to hold one item of "additional data". "Additional data" is information that is not currently part of a record but which may be considered necessary to a proper interpretation of the record. Examples would include the ultimate disposition of an arrest or the fact that an unpaid bill is disputed.

...SADD ...

Number of Characters

39. How many times per year would you expect to add such an item of "additional information" to a record?

$$
\begin{array}{ll}
\text {.. NADY... } & \\
\text { Times/Yr. } & \begin{array}{c}
\text { (Cross Check Only, NADY Computed } \\
\text { By Program) }
\end{array}
\end{array}
$$

40. The number of characters of storage that would be allotted to store the dissenting opinion of a data subject:

$$
\text { ... SCLM... }
$$

Number of Characters

41. The size of an entry in the record usage log. This entry must contain the date and purpose of the access and the identity of the individual and organization making the inquiry:

...\$LQG...

Number of Characters 
42. What retention time do you think is appropriate for record usage information?

(Not a Program Input)

43. The number of computer instructions that would be executed to generate a usage log entry is:

...IWUL....

Number of Instructions

44. The number of computer instructions that would be executed to reference the "next" record in a sequential scan of the entire file is:

$$
\text { ...ISÇN... (Already in Program) }
$$

Number of Instructions

45. The number of computer instructions that would be executed to generate a hardcopy of all the information relevant to a specific individual is (not counting the time needed to locate and access the information):

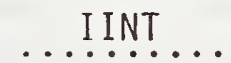

Number of Instructions

46. The amount of computer time required for auditing and program verification purposes is:

...TADT....

Number of Hours 
Section V: Man-hours

These questions deal with the amount of human time, administrative or clerical, required to perform the specified functions.

47. How many man-hours of auditing time per year would be required to assure that the system was in compliance with the Privacy Act?

... HADT ....

Number Hours/Yr.

48. How many man-hours of administrative time would be required to redesign each data collection form to include the appropriate notices to potential data subejcts?

$$
\text { ... HFRM ... }
$$

Hours/Form

49. How many man-hours of administrative time would be needed to develop an appropriate set of security policies?

$$
\text { ... HSPL... }
$$

Hours

50. How many man-hours of administrative time would be needed to evaluate and reach a decision on a data subject's complaint about the validity of his record?

$$
\text { H............. }
$$

Hours

51. How many man-hours of clerical time would be needed to prepare and enter a subject's claim concerning his record?

$$
\text { ... HPCL... }
$$

Hours

52. How inany man-hours of clerical time would be required to generate a notification to a data subject?

....HGS...

Hours 
53. How many man-hours of clerical time would be needed to accept and process a subject's inquiry concerning his record?

.. HINR....

Hours

54. How many man-hours per year would have to be devoted to security related tasks such as guarding equipment and data, and checking personnel identification? (see additional checklist)

$$
\begin{aligned}
& \text {. HGRD. . . } \\
& \text { Hours }
\end{aligned}
$$

55. How long would it take to manually verify the identity of an individual submitting a transaction offline?

$$
\begin{gathered}
\text { HUID } \\
\text { Hours }
\end{gathered}
$$

56. How many man-hours would be required (on the average) to collect. a required iten of "additional data"?

$$
\text { ... HCAD.... }
$$

Hours

57. How many man-hours would be required to reverify the accuracy and timeliness of an old record?

$$
\text { ... HVER ... }
$$

Hours

58. How many hours of training in security and privacy policies and procedures would be required for each person involved in the operation of your system?

$$
\text { ... HTRC...... }
$$

Hours

59. How many hours of training in security and privacy policies and prosecure would be required for each user of the system?

$$
\text { HTRU .... }
$$

Are you responsible for this training? .......... 
Section VI: Programing Time

A number of proposed privacy regulations would appear to require the modification of existing data handiing programs, and in some cases, the creation of entirely new ones. These questions are designed to get at the allount of work required to accomplish this. If your system already includes a capability listed below, please so indicate.

How many man-hours of programming time (including program design, coding, testing and documentation) would be required to:

60. Support the system auditing function:

$$
\begin{gathered}
\text {... PADT ... } \\
\text { Total Hours }
\end{gathered}
$$

61. Enable the operating system to check and confirm the identity of a user:

$$
\text { PUID }
$$

Total Hours

62. Enable the generation of a notice to each data subject:

... PNOT...

Total Hours

63. Permit checking the authorization of each access on the basis of user identification and stated purpose:

PWAC . . .

\section{Total Hours}

64. Include the capability for checking the "verification date" field each time a record is retrieved, and keeping a list of records older than a specified date:

..PCV. . .

Total Hours 
65. Provide for the "subject's claim" field in each record and ensure that it is included with all responses from an individual's record: . PDSL....

Total Hours

66. Implement the usage log, including the creation, storage, and retrieval of usage records:

. . PLOG....

Total Hours

67. Retrieve all of the data relevant to a particular individual: ..PRET....

Total Hours

68. Print an individual's record in "comprehensible" form: ..PINT ....

Total Hours

69. Provide for the adding or removal of entries in a record's access control field?

. PACC....

Total Hours 


\section{Section VII: Counts}

This section asks for estimates of a few miscellaneous numbers.

70. The number of times per year that a new application might be expected to be added to the system is:

...NNUS...

New Applications/Yr.

71. The fraction of mailed requests to data subjects that would have to be repeated because the subject failed to respond the first time is:

...FRPT....

Percent

72. The dollar value of additional hardware required to maintain the usage $\log$ is:

...KLبG ...

Total Dollars

73. The dollar value of additional hardware needed to provide system and data security is:

... KSSEC ...

Total Dollars

74. Does your organization send a regular mailing (at least once a year) to all data subjects?

Yes.ọr. Nọ. (Not a Program Input)

75. What is your estimate of the fraction of the initial programming cost of a system that is expended annually for ongoing maintenance of that system?

. FMNT ... PRGMNT

Percent 
76. What fraction of the data subjects of this system would you expect to refuse permission to have their records used for some new purpose?

$$
\text { .... Fọ.... }
$$

Percent

77. What fraction of the data subjects of this system would you expect to enquire (per year) about the existence and content of their records?

FREI

Percent

78. Of those data subjects enquiring about the contents of their record, what fraction would you expect to enquire about the usage made of the record?

. FRỤI...

Percent

79. Of those data subjects enquiring about the contents of their record, what fraction would you expect to dispute some aspect of the record?

...F.QALI...

Percent

80. What fraction of the disputes concerning an individual's record would you expect to be settled through a review procedure?

FRDS . . .

Percent 


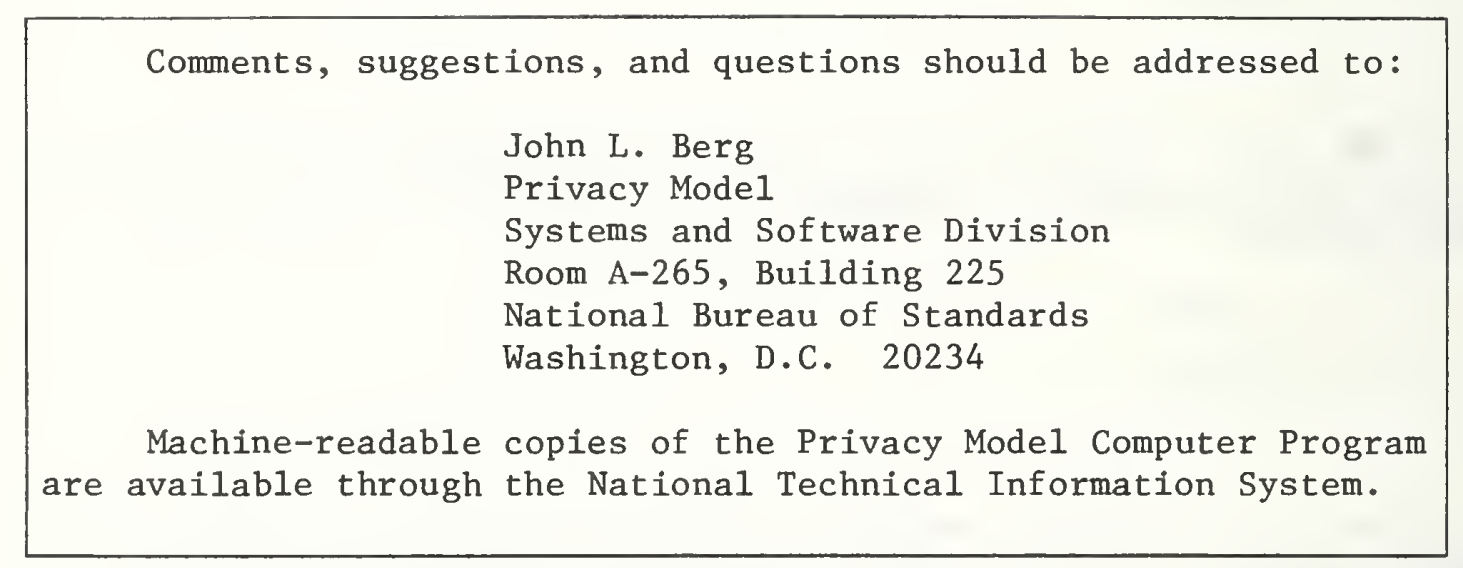


NBS.114A (REV. 7.73 )

\begin{tabular}{|c|c|c|c|}
\hline $\begin{array}{l}\text { U.S. DEPT. OF COMM. } \\
\text { BIBLIOGRAPHIC DATA } \\
\text { SHEET }\end{array}$ & $\begin{array}{l}\text { 1. PUI3LICAATION OR REPORT NO. } \\
\text { NBSIR 76-985 }\end{array}$ & $\begin{array}{l}\text { 2. Gov't Accession } \\
\text { No. }\end{array}$ & 3. Recipient's Accession No. \\
\hline \multirow{2}{*}{\multicolumn{3}{|c|}{$\begin{array}{l}\text { 4. TITLF AND SUBTITI.E } \\
\text { A computer Model to Determine Low } \\
\text { Cost Techniques to Comply with the } \\
\text { Privacy Act of } 1974\end{array}$}} & $\begin{array}{l}\text { 5. Publication Date } \\
\text { February } 1976\end{array}$ \\
\hline & & & 6. Performing Organization Code \\
\hline \multicolumn{3}{|l|}{ 7. $\operatorname{AUTHOR}(S)$} & $\begin{array}{l}\text { 8. Performing Organ. Report No. } \\
\text { D.P. Management Corp. }\end{array}$ \\
\hline \multirow{2}{*}{\multicolumn{3}{|c|}{$\begin{array}{l}\text { 9. PERFORMING ORGANIZATION NAME AND ADDRESS } \\
\text { D.P. Management Corporation } \\
\text { 1 Militia Drive } \\
\text { Lexington, Massachusetts } 02173\end{array}$}} & $\begin{array}{c}\text { 10. Project/Task/Work Unit No. } \\
640.1117\end{array}$ \\
\hline & & & $\begin{array}{l}\text { 11. Contract/Grant No. } \\
5-35935\end{array}$ \\
\hline \multirow{2}{*}{\multicolumn{3}{|c|}{$\begin{array}{l}\text { 12. Sponsoring Organization Name and Complete Address (Street, City, State, ZIP) } \\
\text { National Bureau of Standards } \\
\text { Department of Commerce } \\
\text { Washington, D.C. } 20234\end{array}$}} & $\begin{array}{l}\text { 13. Type of Report \& Period } \\
\text { Covered } \\
\text { Final }\end{array}$ \\
\hline & & & 14. Sponsoring Agency Code \\
\hline
\end{tabular}

15. SUPPLEMENTARY NOTES

16. ABSTRACT (A 200-word or less factual summary of most significant information. If document includes a significant bibliography or literature survey, mention it here.)

This document contains a complete description of the steps necessary to run the Cost of Privacy Model along with a description of the computer program which implements the Model. This document describes the general system requirements for running the program, how to input information to the Model and how to interpret the output.

The Model, which was developed for the National Bureau of Standards by the D.P. Management Corporation of Lexington, Massachusetts, accepts inputs about personal data systems, utilizes algorithms to provide incremental resources required to comply with the Privacy Act of 1974, and applies cost factors to the resources to provide a cost estimate. Cost estimates of alternative compliance techniques can be generated. These can later be compared by the user to determine a cost effective method of compliance.

17. KEY WORDS (six to twelve entries; alphabetical order, capitalize only the first letter of the first key word unless a proper name; separated by semicolons) Computer model; computer security; confidentiality; cost mode1; data security costs; PL-93-579; privacy; Privacy Act of 1974; privacy compliance techniques; privacy costs; privacy model; security costs.

\begin{tabular}{|c|c|c|}
\hline 18. AVAILABILITY $X$ UnIimited & $\begin{array}{l}\text { 19. SECURITY CLASS } \\
\text { (THIS REPURT) }\end{array}$ & 21. NO. OF PAGES \\
\hline$\square$ For Official Distribution. Do Not Release to NTIS & UNCL ASSIF IED & 63 \\
\hline $\begin{array}{l}\square \text { Order From Sup. of Doc., U.S. Government Printing Office } \\
\text { Washington, D.C. 20402, SD Cat. No.C13 }\end{array}$ & $\begin{array}{l}\text { 20. SECURITY CLASS } \\
\text { (THIS PAGE) }\end{array}$ & 22. Price \\
\hline $\begin{array}{l}\text { X] Order From National Technical Information Service (NTIS) } \\
\text { Springfield, Virginia } 22151\end{array}$ & UNCLASSIFIED & $\$ 4.50$ \\
\hline
\end{tabular}




,





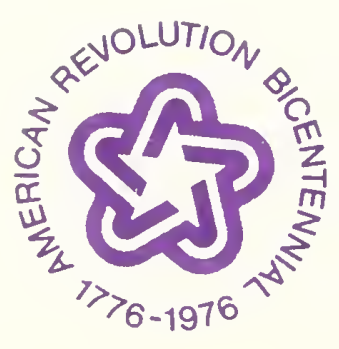

